\title{
Voriconazole in the management of nosocomial invasive fungal infections
}

\author{
Javier Pemán' \\ Miguel Salavert ${ }^{2}$ \\ Emilia Cantón ${ }^{3}$ \\ Isidro Jarque ${ }^{4}$ \\ Eva Romás \\ Rafael Zaragoza ${ }^{6}$ \\ Ángel Viudes ${ }^{7}$ \\ Miguel Gobernado'
}

'Microbiology Department, Hospital Universitario La Fe, Valencia, Spain; 'Infectious Diseases Unit, Hospital Universitario La Fe, Valencia, Spain ${ }^{3}$ Experimental Microbiology Unit, Hospital Universitario La Fe, Valencia, Spain; ${ }^{4}$ Hematology Department, Hospital Universitario La Fe, Valencia, Spain; ${ }^{5}$ Pharmacy Department, Hospital Universitario La Fe, Valencia, Spain; ${ }^{6}$ Intensive Care Unit, Hospital Universitario Dr. Peset, Valencia, Spain; ${ }^{7}$ Medical Department, Pfizer, Madrid, Spain
Correspondence: Javier Pemán Unidad de Micología, Servicio de Microbiología, Hospital Univ La Fe,Av. Campanar, 21, 46009 Valencia, Spain

Tel +34 961973333

Fax +34961973177

Email peman_jav@gva.es

\begin{abstract}
Voriconazole is a new triazole developed for the treatment of life-threatening fungal infections. The drug is available for both oral and intravenous administration; the oral formulation has excellent bioavailability. The side-effect profile of voriconazole presents an acceptable safety and tolerability spectrum: transient visual disturbances, liver enzyme abnormalities, and skin rashes are the most frequently reported side effects but rarely lead to discontinuation. The potential for drug-drug interactions is high, because of its extensive hepatic metabolism. Careful attention to dosage is required, and serum levels and the effects of interacting drugs should be monitored. Review of 25470 isolates of yeasts and 3216 isolates of filamentous fungi showed voriconazole to have broad-spectrum activity against pathogenic yeasts including intrinsically fluconazole-resistant isolates such as Candida krusei, dimorphic fungi, and opportunistic moulds like Aspergillus spp, amphotericin-B-resistant Aspergillus terreus, Fusarium spp, and Scedosporium apiospermum. It displays excellent clinical efficacy in patients with fluconazole-resistant and -susceptible Candida infections, invasive bone and central nervous system aspergillosis, and various refractory fungal infections. Voriconazole has been approved by the US Food and Drug Administration and by the European Medicines Agency for the treatment of invasive aspergillosis, serious infections caused by Fusarium and $S$. apiospermum, fluconazole-resistant invasive Candida infections, and candidemia in nonneutropenic patients.
\end{abstract}

Keywords: voriconazole, azole, mycoses, treatment, yeasts, moulds

\section{Background}

Over the past few decades, the number of patients at risk of developing a serious fungal infection has increased dramatically because of the more potent immunosuppressant therapies used for transplantation, cancer, and other medical conditions, and the rising number of patients with additional risk factors (central venous catheters, broad-spectrum antibiotics, or aggressive surgery), together with the AIDS epidemic. However, despite the introduction in the early 1990s of fluconazole $\left(\right.$ Diflucan $^{\circledR}$, Pfizer Inc, New York, NY, USA), itraconazole (Sporanox ${ }^{\circledR}$, Janssen-Cilag Ltd, Beerse, Belgium), and lipid formulations of amphotericin B, mortality from disseminated fungal infections continues to be unacceptably high.

Despite the advantages of these newer drugs over the older antifungal agents (lower toxicity and a broader spectrum of activity), each drug has its own limitations. Fluconazole is not effective against pathogenic moulds such as Aspergillus spp or Fusarium spp; in addition, some non-Candida albicans species such as C. krusei and $C$. glabrata exhibit fluconazole resistance. Itraconazole has a broader spectrum of activity than fluconazole, but its oral bioavailability is unpredictable and the intravenous formulation is not yet available in some countries. Amphotericin B has broad-spectrum activity, but its use is associated with nephrotoxicity and infusionrelated reactions. Although less toxic, the high cost of lipid formulations such as 


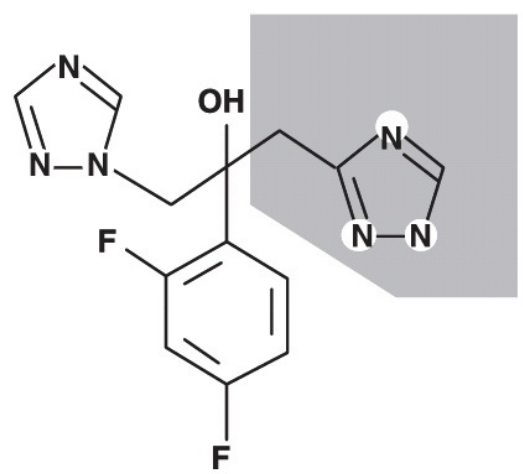

Fluconazole

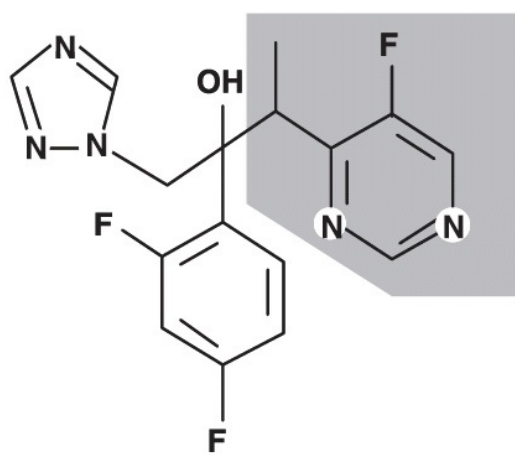

Voriconazole

Figure I Structural formulae of fluconazole and voriconazole.

liposome-encapsulated amphotericin B (Ambisome ${ }^{\circledR}$, Gilead Sciences, Foster City, CA, USA) and amphotericin B-lipid

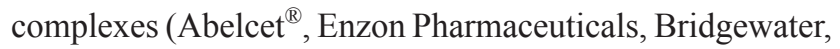
NJ, USA), has limited their practical use.

Voriconazole (Vfend ${ }^{\circledR}$, Pfizer Ltd, New York, NY, USA) belongs to a second generation of triazoles and was developed to fulfill the need for a broad-spectrum antifungal agent with both oral and intravenous formulations. This drug is a synthetic derivative of fluconazole, with fungicidal activity against moulds and excellent pharmacokinetics. It is widely distributed in body fluids, including the cerebral spinal fluid (CSF). It is generally well tolerated, and the reported side effects rarely lead to the drug therapy being discontinued.

Voriconazole was approved by the US Food and Drug Administration (FDA) in May 2002 for the treatment of invasive aspergillosis and serious infections caused by Fusarium spp and Scedosporium apiospermum in cases of intolerance of or refractoriness to other antifungal agents. In November 2003, a license was granted for its use in the treatment of esophageal candidiasis. In December 2004, voriconazole was approved for the treatment of candidemia in nonneutropenic patients, disseminated candidiasis, and Candida infections of the abdomen, kidney, bladder wall, and wounds (FDA 2001). In Europe, voriconazole has been approved by the European Medicines Agency (EMEA) since March 2002 for the treatment of invasive aspergillosis, fluconazole-resistant invasive Candida infections, and serious infections caused by Scedosporium spp and Fusarium spp (EMEA 2002). Recently, in January 2005, the EMEA approved an extension of these indications to include the treatment of candidemia in nonneutropenic patients (EMEA 2005).

\section{Chemistry}

Voriconazole (2R,3S 2-[2,4-diflurophenyl]-3-[5fluropyrimidine-4-yl]-1-[1,2,4-triazol-1-yl] butan-2-ol) is a broad-spectrum triazole antifungal agent with the molecular formula $\mathrm{C}_{16} \mathrm{H}_{14} \mathrm{~N}_{5} \mathrm{OF}_{3}$ and a molecular weight of 349.3 . Voriconazole was developed by the structural modification of fluconazole, with the substitution of a triazole moiety with a fluoropyrimidine ring and the addition of an $\alpha$-methyl group to the propyl backbone (Figure 1) (Sabo and AbdelRahman 2000; Donnelly and de Pauw 2004; Herbrecht 2004).

The addition of the $\alpha$-methyl group increased the affinity of voriconazole for the target enzyme. A fluconazole concentration of $4.8 \mu \mathrm{M}$ is required to inhibit $50 \%$ of the enzyme activity $\left(\mathrm{IC}_{50}\right)$ of Aspergillus fumigatus, whereas only $0.48 \mu \mathrm{M}$ voriconazole is required. Furthermore, the fluoropyrimidine ring increases its antifungal potency, and the addition of fluorine to this ring structure at the 5 position enhances its in vivo efficacy (Sabo and Abdel-Rahman 2000; Herbrecht 2004; Sadaba et al 2004).

Voriconazole is a white to light-colored powder and its solutions $(1 \mathrm{mg} / \mathrm{mL})$ can be made up in dimethyl sulfoxide (DMSO) for animal experiments. The drug is available for oral administration as tablets $(50 \mathrm{mg}$ or $200 \mathrm{mg}$ ) and powder for oral suspension $(40 \mathrm{mg} / \mathrm{mL})$. Voriconazole has limited aqueous solubility. Therefore, the intravenous formulation is combined with solubilizing sulfobutyl ether $\beta$-cyclodextrin sodium (SBECD). This agent is pharmacologically inert, does not affect the pharmacokinetics of voriconazole, and is renally cleared at a constant rate by glomerular filtration (Pearson et al 2003; Herbrecht 2004). 


\section{Pharmacodynamics and pharmacokinetics}

Its structural characteristics provide voriconazole with fungicidal activity against Aspergillus spp and other moulds. Like other azole drugs, voriconazole exerts its mechanism through the inhibition of the fungal cytochrome P450dependent enzyme lanosterol 14- $\alpha$-demethylase (CYP51). A critical step is thus inhibited in the sterol biosynthesis pathway necessary for the production of a functional fungal membrane, as well as sustained growth. Voriconazole also suppresses 24-methylene dihydrolanasterol demethylation in some yeasts and filamentous fungi, explaining in part its activity against fluconazole-resistant moulds (Sabo and Abdel-Rahman 2000; Chandrasekar and Manavathu 2001; Sadaba et al 2004).

The doses recommended for adults by FDA-labeled indication are shown in Table 1 . The intravenous regimen consists of loading doses of $6 \mathrm{mg} / \mathrm{kg}$ bid for the first day, followed by a maintenance dose of $4 \mathrm{mg} / \mathrm{kg}$ bid. Patients may be switched to a maintenance dose of oral voriconazole of $200 \mathrm{mg}$ ( $\geq 40 \mathrm{~kg}$ bodyweight) or $100 \mathrm{mg}(<40 \mathrm{~kg}$ bodyweight) every 12 hours. Oral doses (tablets and suspension) should be taken at least 1 hour before or after meals, because food reduces the rate and extent of absorption (bioavailability reduced by $22 \%$ ). Reductions in maximum concentration $\left(\mathrm{C}_{\max }\right)$ of $34 \%$ and in the area under the curve (AUC) of $24 \%$ have been reported after a high-fat meal.
Both oral and intravenous maintenance doses can be increased by $50 \%$ in patients with refractory disease (Muijsers et al 2002; Donnelly and de Pauw 2004; Herbrecht 2004; Klasko 2005).

In adults, voriconazole displays nonlinear pharmacokinetics, with a greater than proportional increase in plasma concentration with dose escalation and a twocompartment model of distribution. This is thought to be related to its saturable, first-pass metabolism and reduced systemic clearance. Voriconazole displays good oral bioavailability and widespread tissue penetration, with hepatic metabolism and renal excretion of its metabolites (Jeu et al 2003; Johnson and Kauffman 2003; Pearson et al 2003). Unlike some triazole agents, absorption of voriconazole is not affected by gastric $\mathrm{pH}$. The comparative pharmacokinetic characteristics of voriconazole are summarized in Table 2.

In healthy subjects, a 2.5 -fold and a 2.3 -fold increase in the AUC occur when an oral dose of $200 \mathrm{mg}$ bid is increased to $300 \mathrm{mg}$ bid or an intravenous dose of $3 \mathrm{mg} / \mathrm{kg}$ bid is increased to $4 \mathrm{mg} / \mathrm{kg}$ bid, respectively (Klasko 2005). An analysis of 10 clinical trials $(n=280)$ detected no direct relationship between mean, maximum, or minimum plasma voriconazole levels and efficacy. Furthermore, most opportunistic fungal pathogens are inhibited in vitro by $0.5 \mathrm{mg} / \mathrm{L}$ or less, which can usually be achieved with doses of $3-6 \mathrm{mg} / \mathrm{kg}$ bid intravenously or $200 \mathrm{mg}$ bid orally (Radford et al 1997; Espinel-Ingroff 1998).

Table I Recommended voriconazole doses for adults (Klasko 2005)

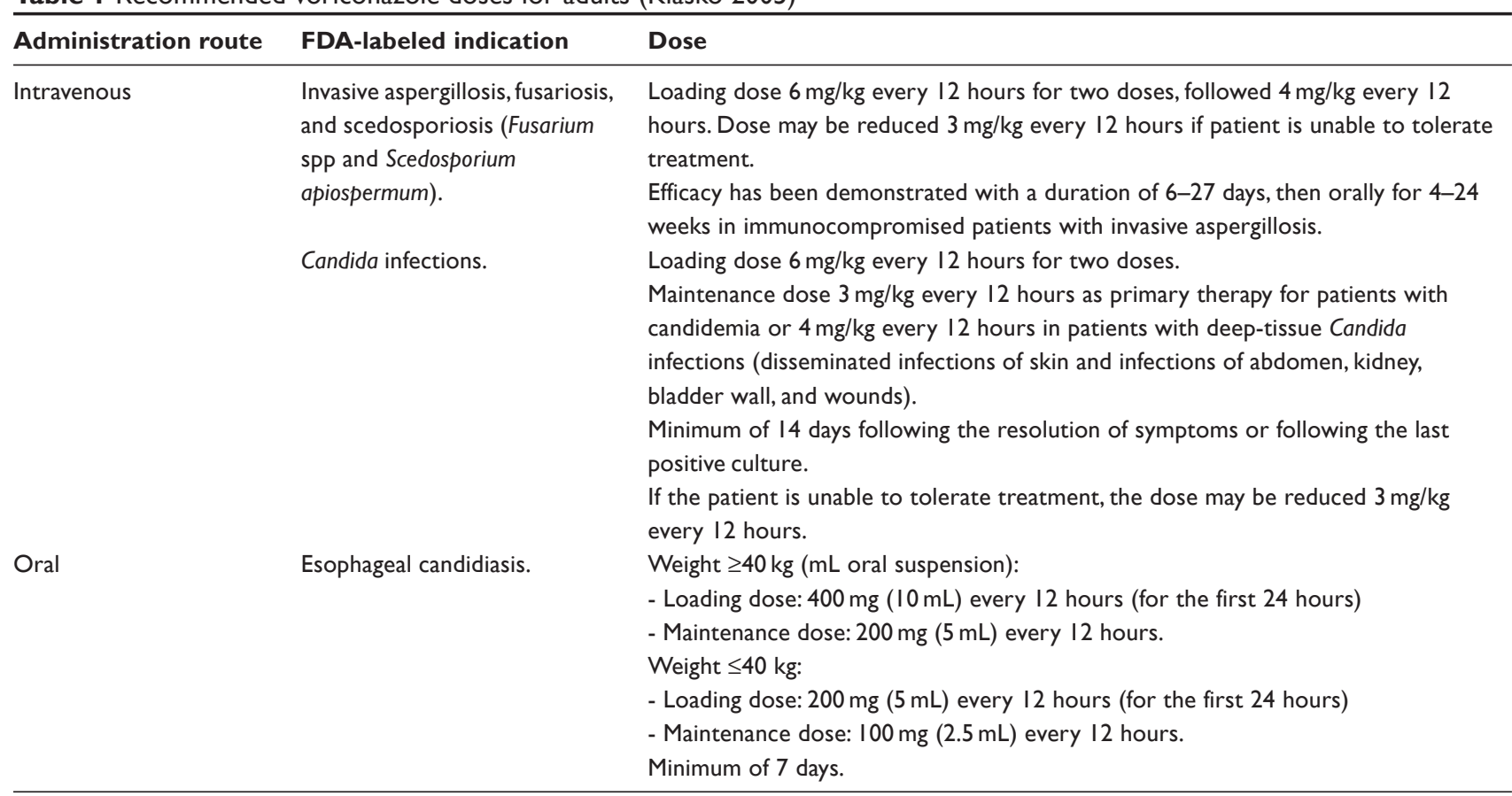

Abbreviations: FDA, US Food and Drug Administration. 
Table 2 Comparative pharmacokinetic properties of triazoles (Sabo and Abdel-Rahman 2000; Jeu et al 2003; Boucher et al 2004; Herbrecht 2004; Sadaba et al 2004; Klasko 2005)

\begin{tabular}{|c|c|c|c|}
\hline & Voriconazole & Itraconazole & Fluconazole \\
\hline $\mathrm{C}_{\max }(\mathrm{mg} / \mathrm{L})$ & $0.9-2.5$ & $0.2-1.1$ & 2 \\
\hline \multirow[t]{3}{*}{$t_{\max }(h)$} & $\mathrm{I}-2$ & $3-4$ & $1-3$ \\
\hline & Oral: suggestive of saturable & & \\
\hline & first-pass metabolism. & & \\
\hline $\operatorname{AUC}(\mu g \times h / m L)$ & Oral 19.86-50.32 & Oral 22.6 & Oral $10.5-15$ \\
\hline Bioavailability (\%) & $90-96$ & $55^{\mathrm{a}}$ & 93 \\
\hline \multirow[t]{4}{*}{ Effects of food } & $\mathrm{C}_{\max }$ reduced by $34 \%$. & Capsules enhanced absorption. & None \\
\hline & AUC reduced by $24 \%$ & Oral solution decreased & \\
\hline & Tablets should be taken on an & bioavailability, so it should & \\
\hline & empty stomach. & not be administered with food. & \\
\hline Protein binding (\%) & $5 I-67$ & 99 & $11-12$ \\
\hline $\mathrm{V}_{\mathrm{d}}(\mathrm{L} / \mathrm{kg})$ & $2-4.6$ & $10-11$ & $0.7-1.2$ \\
\hline \multirow[t]{4}{*}{ Metabolism } & Hepatic, by isoenzymes CYP2CI9, & Hepatic, by CYP isoenzymes. & Hepatic, by CYP \\
\hline & CYP2C9, and CYP3A4, primarily via & & isoenzymes. \\
\hline & N-oxidation. & & \\
\hline & $\mathrm{N}$-oxide metabolite inactive (72\%). & & \\
\hline \multirow[t]{3}{*}{ Excretion } & Renal $<2 \%$ UD and $80 \%-83 \%$ & Renal <I\% UD & Renal $80 \%$ UD \\
\hline & metabolite form. & & \\
\hline & Bile primarily as metabolites. & & \\
\hline$t_{1 / 2}(h)$ & 6 & 24 & 31 \\
\hline \multirow[t]{5}{*}{ Hemodialysis } & Not dialyzable & Not dialyzable & Yes \\
\hline & A 4-hour hemodialysis session & & Hemodyalisis for 3 hours \\
\hline & does not remove a sufficient amount & & decreases plasma levels by \\
\hline & voriconazole to warrant dose & & approximately $50 \%$ \\
\hline & adjustment. & & \\
\hline
\end{tabular}

Abbreviations: $\mathrm{C}_{\max }$, maximum concentration; $\mathrm{t}_{\max }$, time to peak concentration (steady state); $\mathrm{AUC}$, area under the curve; $\mathrm{V}_{\mathrm{d}}$, volume of distribution; $\mathrm{t}_{\mathrm{l} / 2}$, elimination half-life; UD, unchanged drug.

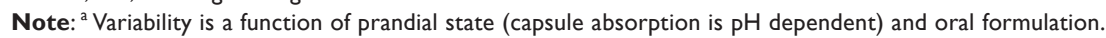

Interindividual variability in plasma voriconazole concentrations is high, whereas intraindividual variation is low. However, plasma levels increase disproportionately with increasing doses, and steady-state levels are reached after 5-7 days with $200 \mathrm{mg}$ bid (Radford et al 1997). With intravenous doses of $6 \mathrm{mg} / \mathrm{kg}$ bid, peak voriconazole plasma levels consistently exceeded $3 \mathrm{mg} / \mathrm{L}$ in a child with invasive aspergillosis, with trough levels maintained above $0.5 \mathrm{mg} /$ L ('t Hek et al 1998).

Cerebral spinal fluid concentrations were $42 \%-67 \%$ of the corresponding plasma levels at various times during treatment (intravenous/oral) in one patient with cerebral aspergillosis (Klasko 2005). In a single nonimmunocompromised patient with fungal empyema, the pleural fluid concentration of voriconazole was $64 \%$ of serum levels when $200 \mathrm{mg}$ bid was administered (Stern et al 2004). Voriconazole has also been detected in the brain, liver, kidney, heart, lung, and spleen at autopsy (Boucher et al 2004).

\section{Dose adjustments in special populations Age}

In clinical trials, median voriconazole plasma concentrations in elderly patients ( $>65$ years) were $\sim 80 \%-90 \%$ higher than those in younger patients. Although the manufacturer does not recommend dose adjustments based on age, voriconazole should be used with caution in elderly patients. There are few pharmacokinetic studies in children, but the available data suggest that voriconazole undergoes linear pharmacokinetics in this population and that children may have a higher capacity to eliminate voriconazole than adults (Jeu et al 2003).

\section{Renal insufficiency}

Voriconazole AUC is not significantly different in patients with moderate renal dysfunction (creatinine clearance 30$50 \mathrm{~mL} / \mathrm{min}$ ). No dose adjustment of the oral formulation is necessary in patients with renal insufficiency. However, moderate renal insufficiency results in the accumulation of 
the intravenous vehicle SBECD, which is renally excreted. In a study of parenteral voriconazole in patients with moderate renal dysfunction, the AUC and $\mathrm{C}_{\max }$ of SBECD increased fourfold and by $50 \%$, respectively, compared with controls (Muijsers et al 2002). The intravenous vehicle of voriconazole is a cyclodextrin. Consequently, in patients with impaired renal function (creatinine clearance $<50 \mathrm{~mL} /$ $\min$ ), assessment of the benefit/risk of administration by an intravenous route is necessary (Johnson and Kauffman 2003).

\section{Hepatic insufficiency}

Dose adjustment is required for patients with chronic hepatic impairment. For patients with Child-Pugh A and B cirrhosis, it is recommended that the standard loading dose be given, followed by half the daily maintenance dose. However, the standard dose is recommended for patients with acute injury resulting from graft-versus-host disease, sinusoidal obstructive syndrome/veno-occlusive disease, or hemodynamic hepatic injury (Johnson and Kauffman 2003; Muijsers et al 2002).

\section{Pregnancy}

Voriconazole is teratogenic in animals and is listed as FDA pregnancy category D (Pearson et al 2003).

\section{Drug interactions}

Voriconazole has many potential interactions because of its extensive hepatic metabolism by CYP2C19, CYP2C9, and CYP3A4. Drugs cleared through the CYP450 system may interact with voriconazole, with complex effects on this microsomal enzyme system exerted by either the target drug or voriconazole. The metabolism of other drugs that are substrates of this metabolic system may be inhibited by voriconazole (Klasko 2005; Muijsers et al 2002; Pearson et al 2003). Recommendations regarding voriconazole drug interactions are shown in Table 3.

\section{Side effects}

Voriconazole is well tolerated. Three specific safety concerns should be considered: adverse visual events, liver function test abnormalities, and skin reactions.

\section{Visual}

Visual disturbances are the most frequent adverse events associated with voriconazole. Transient altered perception of light, photopsia, chromatopsia, photophobia, blurred vision, or color vision changes have been observed in $8 \%$ $44 \%$ of patients (Jeu et al 2003; Johnson and Kauffman 2003; Pearson et al 2003). The visual events begin 30 minutes after administration and last for about 30 minutes. These side effects, associated with higher plasma concentrations and/or doses of the drug, are mild and lead to discontinuation in $<1 \%$ of patients (Johnson and Kauffman 2003; Pearson et al 2003). Abnormalities in electroretinograms persist for the duration of treatment and are completely reversed within two weeks of the discontinuation of therapy (Boucher et al 2004).

\section{Hepatic}

Liver enzyme abnormalities (elevations of alkaline phosphatase, glutamic-oxaloacetic transaminase (GOT)/ aspartate amino transferase (AST), or glutamic-pyruvic transaminase (GPT)/amino alanine transferase (ALT) occur in $12 \%-20 \%$ of patients treated with voriconazole. These side effects usually normalize with continued administration or upon discontinuation of voriconazole ( $4 \%$ to $8 \%$ of patients), but serious events including hepatic failure and death have been reported (Boucher et al 2004). Liver enzyme abnormalities are a dose-limiting adverse effect and may be associated with high voriconazole doses and/or serum concentrations (Jeu et al 2003; Johnson and Kauffman 2003; Pearson et al 2003).

\section{Cutaneous}

Skin rashes were reported in $19 \%$ of patients in clinical studies of voriconazole. Most were mild and rarely led to discontinuation. Several cases of severe skin reactions, including Stevens-Johnson syndrome and toxic epidermal necrolysis, were reported. Because photosensitivity reactions have been described during voriconazole therapy, patients should be advised to avoid sun exposure (Jeu et al 2003; Johnson and Kauffman 2003; Pearson et al 2003).

\section{Miscellaneous}

Other reported side effects of voriconazole include nausea $(5.9 \%)$, vomiting $(4.8 \%)$, headache $(3.2 \%)$, tachycardia $(2.5 \%)$, hypertension (1.9\%), hypotension (1.7\%), abdominal pain (1.7\%), and diarrhea (1.1\%) (Klasko 2005).

\section{In vitro studies}

A large number of studies have been published reporting the in vitro activity of voriconazole against yeasts, yeastlike organisms, and moulds recovered from clinical 
Table 3 Mechanisms of voriconazole drug interactions and recommendations for its use (Muijsers et al 2002; Venkataramanan et al 2002; Johnson and Kauffman 2003; Purkins et al 2003; Groll et al 2004; Klasko 2005)

\begin{tabular}{|c|c|c|c|}
\hline Drug & Mechanism & Results/Drug plasma exposure & Recommendation \\
\hline $\begin{array}{l}\text { Astemizole, } \\
\text { terfenadine, } \\
\text { quinidine, dofetilide }\end{array}$ & $\begin{array}{l}\text { Voriconazole inhibits } \\
\text { CYP3A4 metabolism. }\end{array}$ & $\begin{array}{l}\uparrow \text { plasma concentrations of } \\
\text { astemizole. }\end{array}$ & $\begin{array}{l}\text { Contraindicated. } \\
\text { An increased risk of cardiotoxicity } \\
\text { (QT prolongation, torsade de pointes, cardiac } \\
\text { arrest). }\end{array}$ \\
\hline Azithromycin & $\begin{array}{l}\text { Azithromycin inhibits } \\
\text { CYP450 metabolism of } \\
\text { voriconazole (unclear). }\end{array}$ & $\begin{array}{l}\uparrow \text { voriconazole } C_{\max }(8 \%) \text { and } \\
\operatorname{AUC}(1 \%) .\end{array}$ & No adjustment of voriconazole dose. \\
\hline Barbiturates & $\begin{array}{l}\text { Barbiturates inhibit CYP450 } \\
\text { metabolism of voriconazole. }\end{array}$ & $\begin{array}{l}\text { Systemic exposure to voriconazole } \\
\text { significantly reduced. }\end{array}$ & Contraindicated. \\
\hline Benzodiazepines & $\begin{array}{l}\text { Voriconazole inhibits CYP3A4 } \\
\text { metabolism. }\end{array}$ & $\uparrow$ plasma exposure. & $\begin{array}{l}\text { Frequent monitoring for adverse events and } \\
\text { toxicity (prolonged sedation). Dose adjustment of } \\
\text { benzodiazepine may be necessary. }\end{array}$ \\
\hline $\begin{array}{l}\text { Calcium channel } \\
\text { blockers }\end{array}$ & $\begin{array}{l}\text { Voriconazole inhibits CYP3A4 } \\
\text { metabolism. }\end{array}$ & $\begin{array}{l}\uparrow \text { plasma concentrations of calcium } \\
\text { channel blockers. }\end{array}$ & $\begin{array}{l}\text { Frequent monitoring for adverse events and } \\
\text { toxicity. Dose adjustment of calcium channel } \\
\text { blockers. }\end{array}$ \\
\hline Carbamazepine & $\begin{array}{l}\text { Carbamazepine inhibits } \\
\text { CYP450 metabolism. }\end{array}$ & $\downarrow$ systemic exposure of voriconazole. & Contraindicated. \\
\hline Cyclosporine & $\begin{array}{l}\text { Voriconazole inhibits } \\
\text { CYP3A4 metabolism. }\end{array}$ & $\begin{array}{l}\uparrow \text { AUC cyclosporine } \sim 70 \% \\
\uparrow \text { cyclosporine trough levels by } 2.5 \text {. }\end{array}$ & $\begin{array}{l}\downarrow \text { cyclosporine dose by } 50 \% \text {. Monitor } \\
\text { cyclosporine levels and signs of toxicity. }\end{array}$ \\
\hline Digoxin & $\begin{array}{l}\text { Voriconazole inhibits CYP3A4 } \\
\text { metabolism. }\end{array}$ & $\uparrow$ digoxin $C_{\max }(10 \%)$ and $A U C(I \%)$. & No dose adjustment recommended. \\
\hline Ergot alkaloids & Voriconazole inhibits CYP. & $\begin{array}{l}\text { Likely to be increased (based on } \\
\text { available data; not studied). }\end{array}$ & Contraindicated. \\
\hline Erythromycin & $\begin{array}{l}\text { Erythromycin and } \\
\text { voriconazole inhibit their } \\
\text { CYP3A4 metabolism. }\end{array}$ & $\begin{array}{l}\uparrow \text { plasma concentrations of } \\
\text { voriconazole }\left(C_{\max } 8 \% \text { and } A \cup C \text { I\%) }\right. \\
\text { and erythromycin. }\end{array}$ & $\begin{array}{l}\text { No adjustment of voriconazole dose. } \\
\text { Monitor patients for signs. }\end{array}$ \\
\hline $\begin{array}{l}\text { HMG-CoA } \\
\text { reductase inhibitors } \\
\text { (statins) }\end{array}$ & $\begin{array}{l}\text { Voriconazole inhibits } \\
\text { CYP3A4 metabolism. }\end{array}$ & $\begin{array}{l}\uparrow \text { plasma exposure of HMG-CoA } \\
\text { reductase inhibitors (in vitro } \\
\text { studies). }\end{array}$ & $\begin{array}{l}\text { Frequent monitoring for adverse events and } \\
\text { toxicity related to statins. Increased statin } \\
\text { concentrations in plasma have been associated } \\
\text { with rhabdomyolysis. Adjustment of the statin } \\
\text { dose may be necessary. }\end{array}$ \\
\hline Imatinib & $\begin{array}{l}\text { Voriconazole inhibits } \\
\text { CYP3A4 metabolism. }\end{array}$ & $\uparrow$ plasma exposure of imatinib. & $\begin{array}{l}\text { Monitor for signs of imatinib dose-related adverse } \\
\text { events (fluid retention/water gain, nausea and } \\
\text { vomiting, neutropenia). }\end{array}$ \\
\hline $\begin{array}{l}\text { Indinavir } \\
\text { HIV protease } \\
\text { inhibitors }\end{array}$ & $\begin{array}{l}\text { Indinavir inhibits CYP450 } \\
\text { metabolism of voriconazole. } \\
\text { Voriconazole inhibits } \\
\text { CYP3A4 metabolism of } \\
\text { indinavir. }\end{array}$ & $\begin{array}{l}\uparrow \text { voriconazole } C_{\max }(2 \%) \text { and } \\
A \cup C(7 \%) . \\
\uparrow \text { voriconazole exposure. } \\
\uparrow \text { indinavir } C_{\max }(9 \%) \text { and } A U C \\
(I 1 \%) .\end{array}$ & $\begin{array}{l}\text { No dose adjustment required for indinavir. } \\
\text { Frequent monitoring for adverse events related } \\
\text { to other HIV protease inhibitors. }\end{array}$ \\
\hline NNRTIs & $\begin{array}{l}\text { Voriconazole inhibits CYP3A4 } \\
\text { metabolism. }\end{array}$ & $\uparrow$ plasma exposure (in vitro studies). & $\begin{array}{l}\text { Frequent monitoring for adverse events and } \\
\text { toxicity related to NNRTIs. }\end{array}$ \\
\hline Omeprazole & $\begin{array}{l}\text { Competitive inhibition of } \\
\text { omeprazole and voriconazole } \\
\text { metabolim by CYP2CI9 } \\
\text { and CYP3A4. }\end{array}$ & $\begin{array}{l}\uparrow \text { voriconazole } C_{\max }(15 \%) \text { and } \\
\text { AUC (4I\%). } \\
\uparrow \text { omeprazole } C_{\max } \text { (3.8-fold) } \\
\text { and AUC (2.2-fold). } \\
\uparrow \text { exposure to voriconazole } \\
\text { and omeprazole. }\end{array}$ & $\begin{array}{l}\text { Reduce omeprazole dose by } 50 \% \text { when starting } \\
\text { voriconazole. No change in voriconazole dose. }\end{array}$ \\
\hline Phenytoin & $\begin{array}{l}\text { Voriconazole inhibits } \\
\text { CYP2C9 metabolism of } \\
\text { phenytoin. } \\
\text { Phenytoin induces CYP3A4 } \\
\text { metabolism of voriconazole. }\end{array}$ & $\begin{array}{l}\uparrow \text { phenytoin } C_{\max }(67 \%) \text { and } A \cup C \\
(81 \%) . \\
\downarrow \text { voriconazole } C_{\max }(51 \%) \text { and } \\
A \cup C(31 \%) .\end{array}$ & $\begin{array}{l}\text { Monitor phenytoin levels and phenytoin-related } \\
\text { adverse events. Adjust voriconazole dose to } \\
5 \mathrm{mg} / \mathrm{kg} \text { intravenously or to } 400 \mathrm{mg} \text { orally, twice } \\
\text { daily. }\end{array}$ \\
\hline Prednisolone & $\begin{array}{l}\text { Competitive inhibition of } \\
\text { CYP3A4. }\end{array}$ & $\begin{array}{l}\text { - prednisolone } C_{\max }(1 \mathrm{l} \%) \text { and } \\
\text { AUC (34\%). Slight accumulation } \\
\text { of voriconazole. }\end{array}$ & No dose adjustment required. \\
\hline
\end{tabular}


Table 3 Continued

\begin{tabular}{|c|c|c|c|}
\hline Drug & Mechanism & Results/Drug plasma exposure & Recommendation \\
\hline Rifabutin & $\begin{array}{l}\text { Rifabutin induces CYP450 } \\
\text { metabolism of voriconazole. } \\
\text { Voriconazole inhibits CYP3A4 } \\
\text { metabolism of rifabutin. }\end{array}$ & $\begin{array}{l}\downarrow \text { voriconazole } C_{\max }(66 \%) \text { and } \\
\text { AUC (79\%) } \\
\downarrow \text { rifabutin } C_{\max }(69 \%) \text { and } \\
\text { AUC }(78 \%) .\end{array}$ & $\begin{array}{l}\text { Contraindicated. } \\
\text { If benefits of co-administration outweigh risks, } \\
\text { adjust voriconazole dose to } 5 \mathrm{mg} / \mathrm{kg} \text { intravenously } \\
\text { or to } 400 \mathrm{mg} \text { orally, twice daily. }\end{array}$ \\
\hline Rifampin & $\begin{array}{l}\text { Rifampin induces CYP450 } \\
\text { metabolism of voriconazole. }\end{array}$ & $\begin{array}{l}\downarrow \text { voriconazole } C_{\max }(92 \%) \text { and } \\
\operatorname{A\cup C}(96 \%)\end{array}$ & Contraindicated. \\
\hline Sirolimus & $\begin{array}{l}\text { Voriconazole inhibits } \\
\text { CYP3A4 metabolism. }\end{array}$ & $\uparrow$ plasma concentrations of sirolimus. & Contraindicated. \\
\hline $\begin{array}{l}\text { Sulfonylureas } \\
\text { (tolbutamida, } \\
\text { glipizide, glyburide) }\end{array}$ & $\begin{array}{l}\text { Voriconazole inhibits } \\
\text { CYP3A4 metabolism. }\end{array}$ & $\begin{array}{l}\uparrow \text { plasma concentrations of } \\
\text { sulfonylureas. }\end{array}$ & $\begin{array}{l}\text { Frequent monitoring of blood glucose and } \\
\text { appropriate adjustment of the sulfonylurea dose. }\end{array}$ \\
\hline Tacrolimus & $\begin{array}{l}\text { Voriconazole inhibits } \\
\text { CYP3A4 metabolism } \\
\text { (dose dependent). }\end{array}$ & $\begin{array}{l}\uparrow \text { tacrolimus } C_{\max }(2.2 \text {-fold }) \text { and } \\
\text { AUC (3.2-fold). }\end{array}$ & $\begin{array}{l}\text { Reduce tacrolimus dose by a third when starting } \\
\text { voriconazole. } \\
\text { Monitor plasma levels frequently. }\end{array}$ \\
\hline $\begin{array}{l}\text { Vinca alkaloids } \\
\text { (vincristine, } \\
\text { vinblastine, } \\
\text { vinorelbine) }\end{array}$ & $\begin{array}{l}\text { Voriconazole inhibits } \\
\text { CYP3A4 metabolism. }\end{array}$ & $\begin{array}{l}\uparrow \text { plasma concentrations of } \\
\text { vinca alkaloids. }\end{array}$ & Dose adjustment of vinca alkaloids. \\
\hline $\begin{array}{l}\text { Warfarin oral } \\
\text { anticoagulants }\end{array}$ & $\begin{array}{l}\text { Voriconazole inhibits } \\
\text { CYP3A4 metabolism. }\end{array}$ & $\begin{array}{l}\uparrow \text { warfarin effect } \\
\text { ( } \uparrow \text { prothrombin time). }\end{array}$ & $\begin{array}{l}\text { Monitor prothrombin time. } \\
\text { Adjust warfarin dose if necessary. } \\
\text { Increased risk of bleeding. }\end{array}$ \\
\hline
\end{tabular}

Abbreviations: NNRTIs, nonnucleoside reverse transcriptase inhibitor; $\mathrm{AUC}$, area under the curve; $\mathrm{C}_{\max }$, maximum concentration; $\mathrm{CY}$, cytochrome.

specimens. The majority of authors have evaluated the in vitro activity of voriconazole with reference to the method of the Clinical Laboratory Standards Institute (CLSI) (formerly the National Committee for Clinical Laboratory Standards [NCCLS]) M27-A2 or European Committee on Antimicrobial Susceptibility Testing (EUCAST) methods for yeasts, and M38-A for moulds. Other studies have used marketed methods such as Sensititre YeastOne ${ }^{\circledR}$ (TREK Diagnostic Systems Ltd., West Sussex, England) and Etest ${ }^{\circledR}$ (AB BIODISK, Solna, Sweden) to determine the in vitro susceptibility to voriconazole of pathogenic fungi. The correlation between the results obtained by those methods and those obtained with the CLSI methods is $>90 \%$ for both yeasts and filamentous fungi (Espinel-Ingroff and Rezusta 2002; Espinel-Ingroff et al 2004; Serrano et al 2004; Linares, Charriel, et al 2005). Tentative interpretive breakpoints for voriconazole have recently been established by the CLSI (Minutes of CLSI Antifungal Subcommittee Meeting, 2005), in which a strain is categorized as "susceptible" if the minimal inhibitory concentration (MIC) is $\leq 1 \mu \mathrm{g} / \mathrm{mL}$, "susceptible dose-dependent" if the $\mathrm{MIC}=2 \mu \mathrm{g} / \mathrm{mL}$, and "resistant" if the MIC is $\geq 4 \mu \mathrm{g} / \mathrm{mL}$ (Espinel-Ingroff et al 2005; Pfaller, Boyken, et al 2005).

The in vitro activity of voriconazole has been reviewed in published studies, examining a total of 25470 clinical isolates of yeasts, 104 algae, and 3216 filamentous fungi (Tables 4 and 5).

\section{In vitro activity against yeasts and algae}

A review of in vitro voriconazole activity against yeasts includes 25470 clinical isolates belonging to 15 genera and 60 different species (Table 4). Voriconazole has shown activity against Candida spp, including fluconazole-resistant isolates and those species intrinsically resistant to fluconazole (C. krusei, C. norvegensis, and C. inconspicua). Its in vitro activity against Cryptococcus spp is superior to that of flucytosine and fluconazole: $92 \%$ of isolates are susceptible to voriconazole (MIC $\leq 1 \mu \mathrm{g} / \mathrm{mL}$ ) versus $44 \%$ susceptible to flucytosine (MIC $\leq 4 \mu \mathrm{g} / \mathrm{mL}$ ) and $83 \%$ to fluconazole (MIC $\leq 8 \mu \mathrm{g} / \mathrm{mL}$ ) (Pfaller, Messer, et al 2005).

Moreover, voriconazole has in vitro activity against emerging yeast-like pathogens such as Rhodotorula spp (a microorganism resistant to fluconazole and caspofungin) (Diekema et al 2005) and Blastoschizomyces capitatus (formerly Geotricum capitatum). Data on the in vitro susceptibility to antifungal agents of $B$. capitatus are scarce, although decreased susceptibility to fluconazole and flucytosine have been reported, and high levels of susceptibility to voriconazole (MIC range, 0.03-0.5 $\mu \mathrm{g} / \mathrm{mL}$ ) (Girmenia et al 2003). The in vitro activity of voriconazole 
Table 4 In vitro activity of voriconazole against 25470 strains of yeasts and 104 strains of algae

\section{Genus and species}

(No. strains reviewed) MIC MIC $_{50} \quad$ MIC $_{90}$

Arxiozyma (6)

A. telluris FZ-R

Blastoschizomyces (46)

B. capitatus

B. capitatus FZ-R

B. capitatus FZ-S

Candida (22 70I)

C. albicans (97I5)
$0.03-0.5$

$0.03-0.5$

$0.06-2$

$0.015-0.25$

$0.008->16 \quad 0.008-0.5$
0.12

0.25

$0.34^{\mathrm{a}}$

$0.1^{\mathrm{a}}$

$0.015-2$

Chavez et al 1999; Chryssanthou and Cuenca-Estrella 2002;

Cuenca-Estrella et al 2005; Girmenia et al 2000; Linares et al 2004; Marco et al 2003; Ostrosky-Zeichner, Rex, et al 2003; Pelletier et al 2002; Pemán et al 2005; Pfaller, Jones, et al 1999a; Pfaller et al 2002b; Pfaller, Espinel-Ingroff, et al 2004; Pfaller, Messer, et al 2004; Rubio et al 2003; Takakura et al 2004; Uzun et al 2000; Vazquez et al 1997; Yang et al 2004

Cuenca-Estrella et al 2004

Espinel-Ingroff et al 200I

Maxwell et al 2003; Ostrosky-Zeichner, Rex, et al 2003; Pfaller, Messer, et al I999a; Pfaller et al 2002b; Pfaller et al 2003; Pfaller and Diekema 2004; Quindós et al 2000; Rubio et al 2003

Cuenca-Estrella et al 2004

Espinel-Ingroff et al 200 I; Maxwell et al 2003; Pfaller et al 2003; Rubio et al 2003

Barchiesi et al 2004; Burn et al 2004; Chavez et al 1999; Chryssanthou and Cuenca-Estrella 2002; Cuenca-Estrella et al 2005; Drago et al 2004; Linares et al 2004; Marco et al 2003; Ostrosky-Zeichner, Rex, et al 2003; Pai and Jones 2004; Pelletier et al 2002; Pemán et al 2005; Pfaller, Jones, et al 1999a; Pfaller et al 2002b; Pfaller, Espinel-Ingroff, et al 2004; 2004a; Pfaller, Messer, et al 2004; Rubio et al 2003; Swinne et al 2004; Swinne et al 2005; Takakura et al 2004; Tortorano et al 2003; Uzun et al 2000; Vazquez et al 1997; Yang et al 2004

C. glabrata FZ-R

$0.06-16$

$0.72^{\mathrm{a}}$

$0.01-1$

$0.17^{\mathrm{a}}$

C. guilliermondii (35I)

$0.007->16 \quad 0.06-0.125$

$0.12-0.5$

C. guilliermondii FZ-R

$0.03-2$

$0.22^{\mathrm{a}}$

$0.01-0.25$

$0.07^{\mathrm{a}}$

$12^{\mathrm{a}}$

C. haemulonii FZ-R

C. haemulonii FZ-S

C. inconspicua (6)

$8-16$

0.12

$0.25-4$

0.01

C. inconspicua FZ-S

C. kefyr (56)

C. krusei $(1,337)$

$0.015-0.12$

$0.008-0.125 \quad 0.015 \quad 0.03-0.06$

$<0.008-4 \quad 0.06-0.5 \quad 0.25-2$
Cuenca-Estrella et al 2004

Chryssanthou and Cuenca-Estrella 2002; Maxwell et al 2003; OstroskyZeichner, Rex, et al 2003; Pfaller et al 2002b; Pfaller et al 2003; Pfaller and Diekema 2004; Rubio et al 2003; Tortorano et al 2003 Cuenca-Estrella et al 2004

Pfaller et al 2003

Cuenca-Estrella et al 2004

Cuenca-Estrella et al 2004; Maxwell et al 2003

Espinel-Ingroff et al 200 I; Maxwell et al 2003; Ostrosky-Zeichner, Rex, et al 2003; Pfaller et al 2003; Uzun et al 2000

Chavez et al 1999; Chryssanthou and Cuenca-Estrella 2002; CuencaEstrella et al 2004; Cuenca-Estrella et al 2005; Drago et al 2004; Linares et al 2004; Marco et al 2003; Maxwell et al 2003; OstroskyZeichner, Rex, et al 2003; Pelletier et al 2002; Pemán et al 2005; Pfaller et al 2002b; Pfaller et al 2003; Pfaller, Espinel-Ingroff, et al 2004; Pfaller and Diekema 2004; Pfaller, Messer, et al 2004; Rubio et al 2003; Swinne et al 2004; Swinne et al 2005; Takakura et al 2004; Tortorano et al 2003; Uzun et al 2000;Vazquez et al 1997; Yang et al 2004 Espinel-Ingroff et al 200 I; Pfaller et al 2003 
Table 4 Continued

Genus and species (No. strains reviewed)

C. lipolytica (24)

C. Iusitaniae (582)

C. Iusitaniae FZ-R

C. Iusitaniae FZ-S

C. norvegensis (2)

C. parapsilosis (2703)
0.12

$0.01-0.03$

0.12

\section{MIC}

$0.008-4$

$0.008-0.03$

$\begin{array}{llll}\text { C. parapsilosis FZ-R } & 0.12-0.5 & & 0.25^{\mathrm{a}} \\ \text { C. parapsilosis FZ-S } & 0.01-0.12 & & 0.02^{\mathrm{a}} \\ \text { C. pelliculosa (38) } & 0.06-0.5 & 0.12 & 0.25-0.5 \\ & & & \\ \text { C. rugosa (52) } & 0.008-0.25 & 0.06 & 0.12-0.25 \\ & & & \\ \text { C. rugosa FZ-R } & 0.25 & & 0.25^{\mathrm{a}} \\ \text { C. rugosa FZ-S } & 0.01-0.25 & & 0.06^{\mathrm{a}} \\ \text { C. sake (3) } & 0.015-8 & & 0.5 \\ \text { C. sphoerica (I) } & 0.03 & & \\ \text { C. stellatoidea (I) } & 0.125 & & \\ \text { C. tropicalis (I850) } & 0.008->16 & 0.03-0.5 & 0.03->16\end{array}$

$\begin{array}{llll}\text { C. tropicalis FZ-R } & 0.0 \mathrm{I}-16 & & 0.84^{\mathrm{a}} \\ \text { C. tropicalis FZ-S } & 0.0 \mathrm{I}-4 & & 0.03^{\mathrm{a}} \\ \text { C. zeylanoides FZ-R } & 0.12-0.25 & & 0.18^{\mathrm{a}} \\ \text { C. zeylanoides FZ-S } & 0.008-0.25 & & \\ \text { Candida spp (I88) } & 0.008-2 & 0.03-0.25 & 0.025-2\end{array}$

\section{Cryptococcus (2368)}

C. albidus FZ-R

$0.25-4$

0.25

C. albidus FZ-S

C. gatii FZ-R

C. gatii FZ-S

C. laurentii FZ-R

C. laurentii FZ-S

C. neoformans (2349)

C. neoformans-Africa (52)

C. neoformans-Asia (I I0)

C. neoformans FZ-R

0.12
$0.01^{\mathrm{a}}$
$0.015-4$

$0.008-0.06 \quad 0.015-4$
MIC $_{90}$

$0.015-4$

Espinel-Ingroff et al 200I; Maxwell et al 2003; Ostrosky-Zeichner, Rex, et al 2003; Pelletier et al 2002; Pfaller et al 2003;

Tortorano et al 2003

Chryssanthou and Cuenca-Estrella 2002; Favel et al 2004; Linares et al 2004; Maxwell et al 2003; Ostrosky-Zeichner, Rex, et al 2003;

Pelletier et al 2002; Pfaller et al 2002b; Pfaller et al 2003; Pfaller,

Espinel-Ingroff, et al 2004; Pfallerand Diekema 2004; Pfaller, Messer, et al 2004; Rubio et al 2003; Swinne et al 2004; Swinne et al 2005; Tortorano et al 2003

Cuenca-Estrella et al 2004

Maxwell et al 2003; Pfaller et al 2003

Chavez et al 1999; Chryssanthou and Cuenca-Estrella 2002; Cuenca-Estrella et al 2005; Linares et al 2004; Marco et al 2003; Ostrosky-Zeichner, Rex, et al 2003; Pelletier et al 2002; Pemán et al 2005; Pfaller, Jones, et al 1999a; Pfaller et al 2002b; Pfaller, EspinelIngroff, et al 2004; Pfaller, Messer, et al 2004; Rubio et al 2003; Swinne et al 2004; Swinne et al 2005; Takakura et al 2004; Tortorano et al 2003; Uzun et al 2000; Vazquez et al 1997; Yang et al 2004

Cuenca-Estrella et al 2004

Maxwell et al 2003; Pfaller et al 2003; Pfaller, Messer, et al 2004; Tortorano et al 2003

Espinel-Ingroff et al 200I; Maxwell et al 2003; Ostrosky-Zeichner, Rex, et al 2003; Pfaller et al 2003; Pfaller and Diekema 2004

Cuenca-Estrella et al 2004

Pelletier et al 2002; Pfaller et al 2003

Ostrosky-Zeichner, Rex, et al 2003

Espinel-Ingroff et al 200I

Chavez et al 1999; Chryssanthou and Cuenca-Estrella 2002; CuencaEstrella et al 2005; Linares et al 2004; Marco et al 2003; OstroskyZeichner, Rex, et al 2003; Pelletier et al 2002; Pemán et al 2005; Pfaller, Jones, et al 1999a; Pfaller et al 2002b; Pfaller, Espinel-Ingroff, et al 2004; Pfaller, Messer, et al 2004; Rubio et al 2003; Swinne et al 2004; Swinne et al 2005; Takakura et al 2004; Tortorano et al 2003; Uzun et al 2000; Vazquez et al 1997; Yang et al 2004

Cuenca-Estrella et al 2004

Cuenca-Estrella et al 2004

Maxwell et al 2003; Pfaller et al 2003

Chryssanthou and Cuenca-Estrella 2002; Cuenca-Estrella et al 2004; Marco et al 2003; Pfaller, Jones, et al 1999a; Rubio et al 2003; CuencaEstrella et al 2005; Pfaller, Espinel-Ingroff, et al 2004; Takakura et al 2004

Cuenca-Estrella et al 2004

$0.62^{\mathrm{a}}$

$0.08^{\mathrm{a}}$

$1.25^{\mathrm{a}}$

$0.12^{\mathrm{a}}$

0.12-0.25 Pfaller, Messer, et al 2004; Pfaller, Messer, et al 2005

$0.125 \quad$ Chandenier et al 2004

$0.24^{\mathrm{a}}$

Cuenca-Estrella et al 2004 
Table 4 Continued

\begin{tabular}{|c|c|c|c|c|}
\hline $\begin{array}{l}\text { Genus and species } \\
\text { (No. strains reviewed) }\end{array}$ & MIC & $\mathrm{MIC}_{50}$ & $\mathrm{MIC}_{90}$ & \\
\hline C. neoformans FZ-S & $0.0 \mathrm{I}-0.5$ & & $0.11^{\mathrm{a}}$ & \\
\hline \multicolumn{5}{|l|}{ Debaryomyces (15) } \\
\hline D. hansenii FZ-R & $0.016-8$ & & $0.3 \mathrm{I}^{\mathrm{a}}$ & Cuenca-Estrella et al 2004 \\
\hline D. hansenii FZ-S & $0.016-0.12$ & & $0.03^{\mathrm{a}}$ & \\
\hline \multicolumn{5}{|l|}{ Geotrichum (9) } \\
\hline G. candidum FZ-R & $0.5-4$ & & $1.22^{\mathrm{a}}$ & Cuenca-Estrella et al 2004 \\
\hline G. candidum FZ-S & $0.03-0.25$ & & $0.08^{\mathrm{a}}$ & \\
\hline \multicolumn{5}{|l|}{ Hansenula (5) } \\
\hline H. anomala & $0.12-0.25$ & 0.25 & $0.25^{\mathrm{a}}$ & Espinel-Ingroff et al 200I \\
\hline \multicolumn{5}{|l|}{ Malassezia (70) } \\
\hline M. furfur (24) & $<0.03-0.12$ & & 0.06 & Garau et al 2003 \\
\hline M. pachydermatis (10) & $<0.03-0.12$ & & 0.06 & \\
\hline M. sloffiae (15) & $<0.03$ & & $<0.03$ & \\
\hline M. sympodialis (2I) & $<0.03$ & & $<0.03$ & \\
\hline \multicolumn{5}{|l|}{ Pichia (9) } \\
\hline P. anomala FZ-R & 0.12 & & & Cuenca-Estrella et al 2004 \\
\hline P. anomala FZ-S & 0.06 & & & \\
\hline P. membranifaciens FZ-R & $0.016-0.25$ & & $0.09^{\mathrm{a}}$ & \\
\hline P. norvegensis FZ-R & $0.25-0.5$ & & $0.35^{\mathrm{a}}$ & \\
\hline P. norvegensis FZ-S & 0.01 & & & \\
\hline \multicolumn{5}{|l|}{ Rhodotorula (84) } \\
\hline R. glutinis $(3 \mathrm{I})$ & $<0.06-4$ & & 8 & Diekema et al 2005 \\
\hline R. glutinis FZ-R & $0.25-0.5$ & & $0.37^{\mathrm{a}}$ & Cuenca-Estrella et al 2004 \\
\hline R. mucilaginosa (rubra) (53) & $<0.06-6$ & 4 & 8 & Diekema et al 2005; Espinel-Ingroff et al 200 I \\
\hline R. mucilaginosa FZ-R & $0.25-8$ & & $2.7^{\mathrm{a}}$ & Cuenca-Estrella et al 2004 \\
\hline \multicolumn{5}{|l|}{ Saccharomyces (15) } \\
\hline S. cerevisiae & $0.06-0.25$ & 0.12 & & Espinel-Ingroff et al 200I \\
\hline S. cerevisiae FZ-R & $0.25-2$ & & $0.79^{\mathrm{a}}$ & Cuenca-Estrella et al 2004 \\
\hline S. cerevisiae FZ-S & $0.06-0.12$ & & $0.07^{\mathrm{a}}$ & \\
\hline \multicolumn{5}{|l|}{ Sporobolomyces (3) } \\
\hline S. salmonicolor & $0.25-4$ & & & Espinel-Ingroff et al $200 \mathrm{I}$ \\
\hline \multicolumn{5}{|l|}{ Torulospora (3) } \\
\hline T. delbrueckii FZ-R & 4 & & $4^{\mathrm{a}}$ & Cuenca-Estrella et al 2004 \\
\hline T. delbrueckii FZ-S & $0.06-0.12$ & & $0.08^{\mathrm{a}}$ & \\
\hline \multicolumn{5}{|l|}{ Trichosporon (I34) } \\
\hline T. asahii (beigelii) (69) & $<0.03-1$ & 0.06 & $0.16^{\mathrm{a}}$ & Espinel-Ingroff et al 200I; McGinnis et al 1998; Paphitou et al 2002 \\
\hline T. asahii FZ-R & $0.25-8$ & & $3.36^{\mathrm{a}}$ & Cuenca-Estrella et al 2004 \\
\hline T. asahii FZ-S & $0.06-1$ & & $0.1^{\mathrm{a}}$ & \\
\hline T.asteroides & $<0.03-0.06$ & & & McGinnis et al 1998 \\
\hline T. brassicae & 0.06 & & & \\
\hline T. coremiforme & 0.06 & & & \\
\hline T. cutaneum FZ-R & 8 & & & Cuenca-Estrella et al 2004; McGinnis et al 1998 \\
\hline T. cutaneum FZ-S & $0.01-0.12$ & & $0.06^{\mathrm{a}}$ & \\
\hline T. dulcitum & $0.25-1$ & & $0.5^{\mathrm{a}}$ & McGinnis et al 1998; Paphitou et al 2002 \\
\hline T. faecalie & 0.12 & & & \\
\hline T.gracile & 0.12 & & & \\
\hline T.inkin & 0.06 & & & \\
\hline T.laibachii & $<0.03$ & & & \\
\hline T.loubieri & 0.12 & & & \\
\hline T. montevideense & 8 & & & \\
\hline T. mucoides FZ-R & $0.03-8$ & & $1.2^{\mathrm{a}}$ & Cuenca-Estrella et al 2004 \\
\hline T. mucoides FZ-S \& SDD & $0.125-0.5$ & & $0.25^{\mathrm{a}}$ & McGinnis et al 1998 \\
\hline T. mucoides FZ-S & $0.01-0.25$ & & $0.09^{\mathrm{a}}$ & Cuenca-Estrella et al 2004 \\
\hline T. ovoides FZ-S & 0.06 & & & McGinnis et al 1998 \\
\hline T.non-asahii & & & 16 & McGinnis et al 1998; Paphitou et al 2002 \\
\hline T.pullulans & I & & & \\
\hline T. sporotrichoides & 0.06 & & & \\
\hline
\end{tabular}


Table 4 Continued

Genus and species

(No.strains reviewed) MIC MIC $_{50} \quad$ MIC $_{90}$

Yarrowia (8)

Y.lipolytica FZ-R

$0.12-2 \quad 0.59^{\mathrm{a}}$

Cuenca-Estrella et al 2004

Y. lipolytica FZ-S

$0.0 \mathrm{I}-0.25 \quad 0.05^{\mathrm{a}}$

Algae (104)

Prototheca wickerhamii

$<0.008-0.5 \quad 0.12$

0.5

Linares, Solis, et al 2005

${ }^{a}$ Geometric mean. Voriconazole activity has been separated according to fluconazole susceptibility when authors presented it in that way.

Abbreviations: FZ-R, fluconazole-resistant (MIC $\geq 64 \mu \mathrm{g} / \mathrm{mL}$ ); FZ-SDD, fluconazole susceptible-dose dependent (MIC = I6-32 $\mu \mathrm{g} / \mathrm{mL}$ ); FZ-S, fluconazole-susceptible $(\mathrm{MIC} \leq 8 \mu \mathrm{g} / \mathrm{mL})$; MIC, range of minimal inhibitory concentrations $(\mu \mathrm{g} / \mathrm{mL})$; $\mathrm{MIC}_{50}$ and $\mathrm{MIC}_{90}$, range of minimal inhibitory concentration at which $50 \%$ and $90 \%$ of isolates are inhibited, respectively.

against Trichosporon depends on the species: among all the species assayed (Table 4), high voriconazole MIC values ( $\geq 4 \mu \mathrm{g} / \mathrm{mL}$ ) were found for some T. asahii and T. mucoides isolates (Pfaller and Diekema 2004; Rodriguez-Tudela et al 2005).

The susceptibility to voriconazole of fluconazoleresistant isolates depends on the phenotype. Isolates with the fluconazole-resistant and itraconazole-susceptible phenotype (RS) are susceptible to voriconazole and those with the fluconazole- and itraconazole-resistant phenotype (RR) are resistant to voriconazole.

Nearly all in vitro studies of voriconazole recorded high MICs $(\geq 4 \mu \mathrm{g} / \mathrm{mL})$ for the species $C$. albicans, $C$. glabrata, and C. tropicalis, and for some of the emerging species of Candida (Table 4), although the majority of authors reported very low $\mathrm{MIC}_{90}$ values for $C$. albicans $(\leq 0.06 \mu \mathrm{g} / \mathrm{mL})$. It is difficult to determine the percentage of voriconazole-resistant isolates for each species because published data refer to the range and $\mathrm{MIC}_{90}$ or the percentage of isolates inhibited by $\leq 1 \mu \mathrm{g} / \mathrm{mL}$. An in vitro study of 6972 invasive clinical Candida spp isolates from more than 200 centers worldwide (Pfaller et al 2002b) reported an overall resistance to voriconazole of $1 \%$, and among the fluconazole-resistant isolates, $71 \%$ were susceptible to voriconazole.

Prototheca wickerhamii is the causal agent of cutaneous and subcutaneous infections and, in rare cases, systemic disease. The in vitro activity of voriconazole against this intrinsically fluconazole-resistant alga has recently been reported. All 104 isolates assayed were inhibited by $\leq 0.5 \mu \mathrm{g} /$ $\mathrm{mL}$ voriconazole (Linares, Solis, et al 2005).

Cross-resistance among triazoles has been reported only for the RR phenotype. Notably, voriconazole is active against both the RR and RS phenotypes of C. krusei (Pfaller et al 2002b), suggesting that the mechanisms of resistance to azoles are different in this species from those of other Candida spp.

\section{Fungicidal activity of voriconazole}

Partial growth inhibition or "trailing" has been reported for $C$. albicans and $C$. tropicalis isolates, whereas voriconazole produces complete inhibition of growth in other species. The minimum fungicidal concentration (MFC) of voriconazole has been determined for these strains and also for C. albicans and C. tropicalis isolates that have no trailing growth. In the first report of voriconazole fungicidal activity against Candida spp, the MFC ranged from $0.03 \mu \mathrm{g} / \mathrm{mL}$ to $2 \mu \mathrm{g} / \mathrm{mL}$ for C. $k r u s e i$, C. parapsilosis, C. dubliniensis, C. lusitaniae, $C$. albicans, and C. tropicalis; the MFC values were $\geq 4 \mu \mathrm{g}$ / $\mathrm{mL}$ against only $C$. glabrata and $C$. dubliniensis (Rubio et al 2005). Moreover, the fungicidal activity of voriconazole against $10 \%$ of non-C. albicans species and $40 \%$ of $C$. parapsilosis has been observed (Ghannoum et al 2005). Other authors have reported voriconazole fungicidal activity to be $\geq 8 \mu \mathrm{g} / \mathrm{mL}$ against Trichosporon spp (Paphitou et al 2002) and C. neoformans (van Duin et al 2004). In contrast, voriconazole fungicidal activity has not been demonstrated with killing curves except for C. neoformans (Manavathu et al 1998; Ernst et al 2002).

\section{In vitro activity against filamentous fungi}

The activity of voriconazole against filamentous fungi has been studied for dimorphic, basidiomycetes, dematiaceus, dermatophytes, zygomycetes, and hyphomycetes moulds (Table 5). In total, the activity of voriconazole against 3216 filamentous fungi has been reviewed. Many of the mould species evaluated in the in vitro susceptibility studies are uncommon causes of human infections, and the number of isolates tested has been limited.

Voriconazole is active against a wide range of dematiaceus moulds, including those that cause eumycetoma, such as Leptosphaeria senegalensis and Madurella mycetomatis. Among the 30 genera tested by 
Table 5 In vitro activity of voriconazole against 3216 strains of filamentous fungi

\begin{tabular}{|c|c|c|c|}
\hline Genus and species (No. tested) & MIC/MFC & $\mathrm{MIC}_{90}$ & Reference \\
\hline \multicolumn{4}{|l|}{ Basidiomycetes (44) } \\
\hline Bjerkandera adusta (14) & $0.25-0.5$ & 0.5 & Gonzalez et al 200I \\
\hline Coprinus spp (8) & $0.25-0.5$ & 0.5 & Gonzalez et al 2001 \\
\hline Schizophyllum commune (5) & $0.5-1$ & 0.5 & Gonzalez et al 200I \\
\hline Unidentified (I7) & $0.5-1$ & 0.5 & Gonzalez et al 200I \\
\hline \multicolumn{4}{|l|}{ Dematiaceus (536) } \\
\hline Alternaria alternata (5) & $0.5-1$ & $0.63^{b}$ & Espinel-Ingroff et al 200I; Radford et al 1997 \\
\hline Alternaria spp (4) & $\mathrm{I}-2$ & $1.25^{\mathrm{b}}$ & Espinel-Ingroff et al $2001 \mathrm{I}$ \\
\hline Aurebasidium pullulans (5) & $0.03-0.25$ & $0.14^{\mathrm{b}}$ & Espinel-Ingroff et al 200I \\
\hline Bipolaris australiensis (6) & $0.125-2$ & $0.2^{\mathrm{b}}$ & Espinel-Ingroff et al 200I; Radford et al 1997 \\
\hline Bipolaris hawaiiensis (23) & $0.06-0.5$ & $0.15-0.5^{b}$ & Espinel-Ingroff et al 2001 \\
\hline Bipolaris specifera (32) & $0.06-2$ & $0.29-2^{b}$ & Espinel-Ingroff et al $200 \mathrm{I}$ \\
\hline Bipolaris spp (16) & $0.12-1$ & $0.33^{\mathrm{b}}$ & Espinel-Ingroff I998 \\
\hline Botryomyces caespitosus (I) & 2 & & Espinel-Ingroff et al 2001 \\
\hline Chaetomium globosum (4) & $0.125-0.5$ & $0.21^{\mathrm{b}}$ & Espinel-Ingroff et al $200 \mathrm{I}$ \\
\hline Cladophialophora carrionii (22) & $0.03-0.125$ & $0.05^{\mathrm{b}}$ & Espinel-Ingroff et al $200 \mathrm{I}$ \\
\hline Cladophialophora bantiana (44) & $<0.03-1$ & $0.12-0.5$ & Espinel-Ingroff et al 200 I; Radford et al I 997 \\
\hline Cladosporium cladosporioides (3) & $0.06-1$ & $0.08^{\mathrm{b}}$ & Espinel-Ingroff et al 2001 \\
\hline Cladosporium sphaerospermum (5) & $0.5-1$ & $0.87^{b}$ & Espinel-Ingroff et al $200 \mathrm{I}$ \\
\hline Coniothyrium fuckelii (5) & $0.125-0.5$ & $0.22^{\mathrm{b}}$ & Espinel-Ingroff et al $200 \mathrm{I}$ \\
\hline Curvularia inaequalis (I) & 0.25 & & Espinel-Ingroff et al $200 \mathrm{I}$ \\
\hline Curvularia lunata (22) & $0.06-1$ & $0.22^{\mathrm{b}}$ & Espinel-Ingroff et al 200I; Radford et al 1997 \\
\hline Curvularia senegalensis ( 3 ) & $0.06-00.25$ & $0.2^{\mathrm{b}}$ & Espinel-Ingroff et al 2001 \\
\hline Curvularia verruculosa (3) & 0.125 & & Espinel-Ingroff et al $200 \mathrm{I}$ \\
\hline Dactylaria constricta (2I) & $0.03-1 / 0.5->8$ & $0.12-0.5$ & Espinel-Ingroff et al $200 \mathrm{I}$ \\
\hline Dissitumurus exedrus (I) & 0.25 & & Espinel-Ingroff et al $200 \mathrm{I}$ \\
\hline Drechslera biseptata (I) & 0.06 & & Espinel-Ingroff et al 200I \\
\hline Exophiala jeanselmei (36) & $<0.03-8$ & $0.6^{\mathrm{b}}$ & Espinel-Ingroff et al 200I; Radford et al 1997 \\
\hline Exophiala moniliae (3) & $0.25-0.5$ & $0.32^{\mathrm{b}}$ & Espinel-Ingroff et al 2001 \\
\hline Exophiala pisciphila (4) & $0.25-1$ & $0.59^{\mathrm{b}}$ & Espinel-Ingroff et al 200I \\
\hline Exophiala spinifera $(10)$ & $0.125-1$ & $0.2^{\mathrm{b}}$ & Espinel-Ingroff et al $200 \mathrm{I}$ \\
\hline Exserohilum rostratum (I2) & $0.06-0.5$ & $0.17^{b}$ & Espinel-Ingroff et al $200 \mathrm{I}$ \\
\hline Fonsecaea compacta (8) & $<0.03-0.5$ & $0.14^{\mathrm{b}}$ & Espinel-Ingroff et al 200I; Radford et al 1997 \\
\hline Fonsecaea pedrosoi (36) & $<0.03-1 /<0.03-1$ & 0.06 & Espinel-Ingroff et al 200I; Radford et al I 997 \\
\hline Hormonema dematioides (3) & $0.06-032$ & $0.23^{b}$ & Espinel-Ingroff et al $200 \mathrm{I}$ \\
\hline Hortaea spp (II) & $0.03-0.125$ & $0.05^{\mathrm{b}}$ & Espinel-Ingroff et al $200 \mathrm{I}$ \\
\hline Lasiodiploidia theobromae (2) & I & & Radford et al 1997 \\
\hline Lecythophora hoffmanii (5) & $0.125-0.5$ & $0.29^{\mathrm{b}}$ & Espinel-Ingroff et al $200 \mathrm{I}$ \\
\hline Lecythophora mutabilis (6) & $0.125-0.5$ & $0.22^{\mathrm{b}}$ & Espinel-Ingroff et al 200I; Radford et al 1997 \\
\hline Leptosphaeria senegalensis (2) & $<0.03-0.06$ & & Radford et al I 997 \\
\hline Madurella grisea $(\mathrm{I})$ & 0.5 & & Espinel-Ingroff et al 200I \\
\hline Madurella mycetomatis (8) & $0.03-0.5$ & $0.05^{\mathrm{b}}$ & Espinel-Ingroff et al 200I; Radford et al 1997 \\
\hline Phaeoacremonium parasiticum (I8) & $0.125-2 / 0.25-1$ & $0.25^{\mathrm{b}}$ & Espinel-Ingroff et al $200 \mathrm{I}$ \\
\hline Phaeoannellomyces elegans (4) & $0.06-8$ & $0.5^{\mathrm{b}}$ & Espinel-Ingroff et al $200 \mathrm{I}$ \\
\hline Phaeosclera dematioides (I) & 4 & & Espinel-Ingroff et al 200I \\
\hline Phialemonium curvatum (3) & 0.25 & & Espinel-Ingroff et al $200 \mathrm{I}$ \\
\hline Phialemonium obovatum (5) & $0.25-1$ & $0.5^{\mathrm{b}}$ & Espinel-Ingroff et al $200 \mathrm{I}$ \\
\hline Phialophora americana (3) & 0.125 & & Espinel-Ingroff et al $200 \mathrm{I}$ \\
\hline Phialophora fastigiata (I) & I & & Espinel-Ingroff et al 200I \\
\hline Phialophora repens (3) & 0.25 & & Espinel-Ingroff et al 2001 \\
\hline Phialophora richardsiae (II) & $0.25-2$ & $0.64^{\mathrm{b}}$ & Espinel-Ingroff et al 200I \\
\hline Phialophora verrucosa (25) & $0.03-0.5$ & $0.12^{b}$ & Espinel-Ingroff et al $200 \mathrm{I}$ \\
\hline Ramichloridium mackenziei (4) & 0.06 & & Radford et al I 997 \\
\hline Rhinocladiella aquaspersa (2) & $0.03-0.25$ & $0.09^{\mathrm{b}}$ & Espinel-Ingroff et al $200 \mathrm{I}$ \\
\hline Rhinocladiella atrovirens (3) & $0.03-0.5$ & $0.18^{\mathrm{b}}$ & Espinel-Ingroff et al $200 \mathrm{I}$ \\
\hline Scolecobasidium constrictum (5) & $0.03-8$ & $0.43^{\mathrm{b}}$ & Espinel-Ingroff et al 2001 \\
\hline Scolecobasidium humicola (4) & $0.03-4$ & $0.21^{\mathrm{b}}$ & Espinel-Ingroff et al $200 \mathrm{I}$ \\
\hline Scytalidium dimidiatum (24) & $<0.03-0.5$ & $0.1^{b}$ & Espinel-Ingroff et al 200 I; Lacroix and Feuilhade 2005 \\
\hline
\end{tabular}


Table 5 Continued

\begin{tabular}{|c|c|c|c|}
\hline Genus and species (No. tested) & MIC/MFC & $\mathrm{MIC}_{90}$ & Reference \\
\hline Scytalidium hyalinum (I5) & $<0.002-0.006$ & & Lacroix and Feuilhade 2005 \\
\hline Wangiella dermatiditis (3I) & $0.03-0.5 / 0.06-1$ & 0.12 & Espinel-Ingroff et al 200 I; Radford et al 1997 \\
\hline \multicolumn{4}{|l|}{ Dermatophytes (120) } \\
\hline Microsporum canis (34) & $0.06-2$ & 0.12 & Serrano-Martino et al 2003 \\
\hline Microsporum gypseum (I3) & $0.12-1$ & 0.5 & Serrano-Martino et al 2003 \\
\hline Trichophyton mentagrophytes (6I) & $0.06-1$ & 0.5 & Serrano-Martino et al 2003 \\
\hline Trichophyton rubrum (I2) & $0.06-2$ & 0.25 & Serrano-Martino et al 2003 \\
\hline \multicolumn{4}{|l|}{ Dimorphic (532) } \\
\hline Blastomyces dermatitidis (I42) & $<0.03-16 /<0.03-32$ & 0.25 & Espinel-Ingroff et al 200I \\
\hline Coccidioides immitis (142) & $<0.03-0.5 />32$ & 0.25 & Espinel-Ingroff et al 200I \\
\hline Histoplasma capsulatum (144) & $<0.03-2 /<0.03->32$ & 0.25 & Espinel-Ingroff et al $200 \mathrm{I}$ \\
\hline Paracoccidioides brasiliensis (19) & $<0.03-2$ & & Espinel-Ingroff et al 200I \\
\hline Penicillium marneffei (34) & $<0.03$ & & Espinel-Ingroff et al 200I; Radford et al 1997 \\
\hline Sporothrix schenckii (5I) & $0.12->16 / 0.5->16$ & $>16$ & Espinel-Ingroff et al 200I; Radford et al 1997 \\
\hline \multicolumn{4}{|l|}{ Hyphomycetes $(1,922)$} \\
\hline Acremonium alabamense (I) & 0.25 & & Espinel-Ingroff et al 200I \\
\hline Acremonium kiliense $(2)$ & 1 & & Radford et 1997 \\
\hline Acremonium spp (I) & 0.25 & & Marco et al 1998 \\
\hline Acremonium strictum (I) & 2 & & Espinel-Ingroff et al 200I \\
\hline Aspergillus flavipes (2) & $0.03-0.12$ & & Serrano et al 2003 \\
\hline Aspergillus flavus (2 I8) & $<0.03-2 / 0.25-8$ & $0.06-1$ & $\begin{array}{l}\text { Diekema et al 2003; Espinel-Ingroff et al 200I; Espinel-Ingroff } \\
\text { and Rezusta 2002; Hsueh et al 2005; Maesaki et al 2000; Marco } \\
\text { et al I998; Pfaller et al 2002a; Serrano et al } 2003\end{array}$ \\
\hline Aspergillus fumigatus (802) & $<0.03-8 / 0.25->8$ & $0.25-1$ & $\begin{array}{l}\text { Clancy and Nguyen 1998; Cuenca-Estrella et al 1998; Diekema } \\
\text { et al 2003; Espinel-Ingroff et al 200I; Espinel-Ingroff and Rezusta } \\
\text { 2002; Hsueh et al 2005; Maesaki et al 2000; Marco et al 1998; } \\
\text { Pfaller et al 2002a; Serrano et al } 2003\end{array}$ \\
\hline Aspergillus glaucus (8) & $0.06-0.12$ & 0.06 & Serrano et al 2003 \\
\hline Aspergillus nidulans (26) & $0.03-4 / 0.12-4$ & 0.5 & Espinel-Ingroff et al 200I; Espinel-Ingroff and Rezusta 2002 \\
\hline Aspergillus niger (| $4 \mid$ ) & $<0.03-4 / 0.25-2$ & $0.12-2$ & $\begin{array}{l}\text { Diekema et al 2003; Espinel-Ingroff et al 200I; Espinel-Ingroff } \\
\text { and Rezusta 2002; Hsueh et al 2005; Maesaki et al 2000; Marco } \\
\text { et al I998; Pfaller et al 2002a; Serrano et al } 2003\end{array}$ \\
\hline Aspergillus terreus (188) & $<0.03-2 / 1-16$ & $0.25-1$ & $\begin{array}{l}\text { Diekema et al 2003; Espinel-Ingroff et al 200I; Espinel-Ingroff } \\
\text { and Rezusta 2002; Hsueh et al 2005; Marco et al I998; Pfaller et } \\
\text { al 2002a; Serrano et al } 2003\end{array}$ \\
\hline Aspergillus ustus (12) & $0.25-8 / 0.25-32$ & 8 & Espinel-Ingroff et al 200I \\
\hline Aspergillus versicolor (29) & $0.06-2$ & I & Diekema et al 2003; Pfaller et al 2002a \\
\hline Aspergillus spp (32) & $0.12->8$ & 1 & Pfaller et al $2002 a$ \\
\hline Fusarium moniliforme (9) & $0.5->8 / 4->8$ & 2 & Espinel-Ingroff et al 2001 \\
\hline Fusarium oxysporum (33) & $0.25->8 / 2->16$ & 8 & Espinel-Ingroff et al 200I; Minassian et al 2003; Radford et al 1997 \\
\hline Fusarium proliferatum (6) & $\mathrm{I}-2$ & & Espinel-Ingroff et al 200I \\
\hline Fusarium solani $(77)$ & $0.25->16 / 2->16$ & $2->8$ & Espinel-Ingroff et al 200I; Minassian et al 2003; Radford et al 1997 \\
\hline Fusarium verticillioides (3) & $\mathrm{I}-8$ & $\mathrm{I}^{\mathrm{a}}$ & Minassian et al 2003 \\
\hline Fusarium spp (24) & $0.25->8$ & $8->8$ & Diekema et al 2003; Marco et al 1998 \\
\hline Paecilomyces boydii (6) & $0.25-0.5$ & $0.33^{b}$ & Espinel-Ingroff 1998 \\
\hline Paecilomyces lilacinus (8) & $0.12-1 / 1-4$ & 0.5 & Espinel-Ingroff et al 200I; Radford et al 1997 \\
\hline Paecilomyces variotii (2) & $4->64$ & & Radford et al 1997 \\
\hline Paecilomyces spp (7) & $0.03-2$ & & Diekema et al 2003; Marco et al 1998 \\
\hline Scedosporium apiospermum (60) & $0.03-2$ & $0.25-2$ & $\begin{array}{l}\text { Carrillo and Guarro 200I; Cuenca-Estrella et al 1999; Meletiadis } \\
\text { et al 2002; Minassian et al 2003; Radford et al } 1997\end{array}$ \\
\hline Scedosporium prolificans (I54) & $0.06-32 / 2->8$ & $4-16$ & $\begin{array}{l}\text { Carrillo and Guarro 200I; Cuenca-Estrella et al 1999; Espinel- } \\
\text { Ingroff et al 200I; Meletiadis et al 2002; Minassian et al 2003; } \\
\text { Radford et al } 1997\end{array}$ \\
\hline Scopulariopsis brevicaulis (2) & $4-8$ & & Radford et al 1997 \\
\hline Scopulariosis brumptii (3) & $2-8$ & & Espinel-Ingroff et al 200I \\
\hline Pseudallescheria boydii (59) & $0.06-1 / 0.25->16$ & 0.5 & Espinel-Ingroff et al 200I; Marco et al 1998 \\
\hline Trichoderma longibrachiatum (5) & $2 / 4$ & 2 & Espinel-Ingroff et al 200I \\
\hline Thrichoderma spp (I) & 0.25 & & Marco et al 1998 \\
\hline
\end{tabular}


Table 5 Continued

\begin{tabular}{|c|c|c|c|}
\hline Genus and Species (No. tested) & MIC/MFC & $\mathrm{MIC}_{90}$ & Reference \\
\hline \multicolumn{4}{|l|}{ Zygomycetes (I82) } \\
\hline Absidia corymbifera $(20)$ & $4->16 / 16->16$ & $16->16$ & Espinel-Ingroff et al 200I; Minassian et al 2003 \\
\hline Absidia elegans (I) & 16 & 16 & Dannaoui et al 2003 \\
\hline Absidia spp (25) & $2-16$ & 16 & Dannaoui et al 2003; Gil-Lamaignere et al 2005 \\
\hline Apophysomyces elegans (I) & 16 & & McGinnis et al 1998 \\
\hline Cunninghamella bertholletiae (I) & 32 & 32 & Dannaoui et al 2003 \\
\hline Cunninghamella spp (15) & $8->16$ & $>16^{\mathrm{a}}$ & Gil-Lamaignere et al 2005; Minassian et al 2003 \\
\hline Mucor hiemalis (I) & $>32$ & & McGinnis et al 1998 \\
\hline Mucor spp (23) & $I->32$ & $16->32$ & $\begin{array}{l}\text { Dannaoui et al 2003; Diekema et al 2003; Gil-Lamaignere et al } \\
\text { 2005; Minassian et al } 2003\end{array}$ \\
\hline Rhizomucor pusillus (2) & $2-16$ & $5.66^{\mathrm{b}}$ & McGinnis et al 1998 \\
\hline Rhizomucor spp (I0) & 4 & 16 & Dannaoui et al 2003; Minassian et al 2003 \\
\hline Rhizopus arrhizus (23) & $4->32 / 8->16$ & 16 & Espinel-Ingroff et al 200I \\
\hline Rhizopus spp (60) & $I \rightarrow 16$ & $8-16$ & $\begin{array}{l}\text { Dannaoui et al 2003; Diekema et al 2003; Gil-Lamaignere et al } \\
\text { 2005; Marco et al 1998; Minassian et al } 2003\end{array}$ \\
\hline
\end{tabular}

Abbreviations: MIC, range of minimal inhibitory concentrations $(\mu \mathrm{g} / \mathrm{mL})$; MFC, minimal fungicidal concentration $(\mu \mathrm{g} / \mathrm{mL})$; $M I C_{90}$, range of minimal inhibitory concentrations at which $90 \%$ of isolates are inhibited $(\mu \mathrm{g} / \mathrm{mL}){ }^{\text {a }}$, modal value; ${ }^{\mathrm{b}}$, geometric mean.

different authors (a total of 536 isolates), only voriconazole MIC values of $\geq 4 \mu \mathrm{g} / \mathrm{mL}$ were obtained against some isolates of Exophiala jeanselmei, Hormonema dematioides, Phaeoscleria dematioides, Scolecobasidium constrictum, and S. humicola. The highest $\mathrm{MIC}_{90}$ or geometric mean $\mathrm{MIC}$ reported for this group was $1.25 \mu \mathrm{g} / \mathrm{mL}$. Fungicidal activity has also been observed against Fonsecaea pedrosoi, Phaeoacremonium parasiticum, and Wangiella dermatitidis. Furthermore, all 44 basidiomycetes isolates were inhibited by $\leq 1 \mu \mathrm{g} / \mathrm{mL}$ voriconazole (Table 5 ).

The fungicidal activity of voriconazole against the 532 dimorphic fungi reviewed was species dependent. Whereas some isolates of Blastomyces dermatitidis and Sporothrix schenckii have voriconazole MICs of $\geq 16 \mu \mathrm{g} / \mathrm{mL}$, the $\mathrm{MIC}_{90}$ for the majority of species is $0.25 \mu \mathrm{g} / \mathrm{mL}$, and $\geq 16 \mu \mathrm{g} / \mathrm{mL}$ for $S$. schenckii. However, voriconazole showed poor or no in vitro activity against the 182 isolates of zygomycetes evaluated.

Among hyphomycete fungi, voriconazole is active against the most clinically important pathogens (Aspergillus spp, Fusarium spp, and Penicillium marneffei). Moreover, it is effective against the two well-documented itraconazoleresistant $A$. fumigatus (Espinel-Ingroff et al 2002). The $\mathrm{MIC}_{90}$ of voriconazole was usually $\leq 1 \mu \mathrm{g} / \mathrm{mL}$ for most of the Aspergillus spp evaluated. Notably, voriconazole shows good in vitro activity against $A$. terreus, a species resistant to amphotericin B. Nevertheless, the activity of voriconazole against Fusarium spp, Paecilomyces spp, and Scedoporium spp is species dependent, with MICs ranging from $8 \mu \mathrm{g} / \mathrm{mL}$ to $16 \mu \mathrm{g} / \mathrm{mL}$ for non-Fusarium solani species and Paecilomyces variotii (Table 5).

In summary, voriconazole has shown in vitro activity against the most common species of yeast isolates, including both intrinsically and secondarily fluconazole-resistant species. Cross-resistance among triazoles is reported only for the RR phenotype. Notably, voriconazole is active against both RR and RS phenotypes of C. krusei, suggesting different mechanisms of resistance to azoles in this species. Voriconazole also displays activity against most species of filamentous fungi that cause systemic or subcutaneous mycoses, such as Aspergillus spp, S. apiospermum, Fusarium spp, P. marneffei, and most of the dematiaceus moulds. However, voriconazole has poor activity against zygomycetes.

\section{Animal studies}

The efficacy of voriconazole in animal models of invasive infections of Aspergillus spp (pulmonary, disseminated, endocarditic) (George et al 1996; Martin et al 1997; Murphy et al 1997; Chandrasekar et al 2000; Kirkpatrick et al 2000), Scedosporium spp (Capilla et al 2003; Capilla and Guarro 2004), B. dermatitidis (Sugar and Liu 2001), and C. krusei (Ghannoum et al 1999) has been widely studied. In all studies, the efficacy of voriconazole was similar to, or better than, that of the antifungal agent compared (amphotericin $\mathrm{B}$, caspofungin, fluconazole, or itraconazole). 
Table 6 Global response to voriconazole of patients with invasive aspergillosis in clinical studies

\begin{tabular}{|c|c|c|c|c|}
\hline $\begin{array}{l}\text { Nature of } \\
\text { voriconazole } \\
\text { treatment }\end{array}$ & $\begin{array}{l}\text { Total } \\
\text { number of } \\
\text { patients }\end{array}$ & Main underlying disease & Outcome & Reference \\
\hline $\begin{array}{l}\text { Comparative study of } \\
\text { voriconazole vs } \\
\text { amphotericin B }\end{array}$ & 277 & $\begin{array}{l}\text { Allogeneic HSCT, hematological } \\
\text { malignancy }\end{array}$ & $\begin{array}{l}\text { Successful in } 53 \% \\
\text { voriconazole group } \\
\text { vs } 32 \% \text { amphotericin B }\end{array}$ & Herbrecht et al 2002 \\
\hline Salvage therapy & 142 & Hematological malignancy & Satisfactory in $43.7 \%$ & Perfect et al 2003 \\
\hline Primary therapy in $53 \%$ & 116 & Neutropenia, allogeneic HSCT & Successful in $48 \%$ & Denning et al 2002 \\
\hline Salvage therapy in $48 \%$ & 102 & Neutropenia & Successful in $53 \%$ & de Pauw 1999 \\
\hline $\begin{array}{l}\text { Retrospective cohort } \\
\text { study of Aspergillus } \\
\text { terreus invasive infection }\end{array}$ & 83 & $\begin{array}{l}\text { Allogeneic HSCT, hematological } \\
\text { malignancy, SOT, solid tumor }\end{array}$ & Successful in $44.2 \%$ & Steinbach et al 2004 \\
\hline $\begin{array}{l}\text { Retrospective study } \\
\text { of CNS aspergillosis }\end{array}$ & 81 & $\begin{array}{l}\text { Hematological malignancy, } \\
\text { allogeneic HSCT }\end{array}$ & $\begin{array}{l}\text { Complete or partial } \\
\text { response in } 35 \%\end{array}$ & Schwartz et al 2005 \\
\hline Salvage therapy & 51 & $\begin{array}{l}\text { Hematological malignancy, relapsed } \\
\text { malignancy, neutropenia }\end{array}$ & Successful in $41 \%$ & Schlamm et al 2000 \\
\hline $\begin{array}{l}\text { Compassionate or } \\
\text { salvage therapy in } \\
\text { children }\end{array}$ & 42 & $\begin{array}{l}\text { Chronic granulomatous disease, } \\
\text { hematological malignancy }\end{array}$ & Successful in $43 \%$ & Walsh, Lutsar, et al 2002 \\
\hline Open study & 36 & Immunosuppression & Successful in $75 \%$ & Denning et al 1995 \\
\hline Compassionate therapy & 35 & SOT, HSCT, hematological malignancy & $\begin{array}{l}\text { Complete or partial } \\
\text { response in } 53 \%\end{array}$ & Baden et al 2003 \\
\hline $\begin{array}{l}\text { Open-label, } \\
\text { noncomparative, } \\
\text { observational study }\end{array}$ & 33 & Critically ill & Successful in $50 \%$ & Alvarez-Lerma et al 2005 \\
\hline Primary therapy in $50 \%$ & 25 & Nonneutropenia & Successful in $53 \%$ & Dupont et al 1995 \\
\hline $\begin{array}{l}\text { Retrospective study } \\
\text { of bone aspergillosis }\end{array}$ & 20 & Immunosuppression & Successful in $55 \%$ & Mouas et al 2005 \\
\hline Salvage therapy & 24 & Hematological malignancy, HSCT & Successful in $41 \%$ & Green et al 2000 \\
\hline $\begin{array}{l}\text { Retrospective study, } \\
\text { compassionate or } \\
\text { salvage therapy }\end{array}$ & 12 & Hematological malignancy in $52 \%$ & Successful in $66 \%$ & Diaz-Pedroche et al 2005 \\
\hline $\begin{array}{l}\text { Compassionate or } \\
\text { salvage therapy } \\
\text { in children }\end{array}$ & 7 & $\begin{array}{l}\text { Chronic granulomatous disease, } \\
\text { hematological malignancy }\end{array}$ & Successful in $43 \%$ & Cesaro et al 2003 \\
\hline Retrospective study & 4 & Neutropenia & Successful in $75 \%$ & Caillot et al 1997 \\
\hline Salvage therapy & 5 & SOT recipients & Successful in $60 \%$ & Fortun et al 2003 \\
\hline
\end{tabular}

Abbreviations: HSCT, hematopoietic stem-cell transplant; SOT, solid organ transplant.

When the activity of voriconazole was studied in combination with amphotericin $\mathrm{B}$, caspofungin, or micafungin, no antagonism was observed (Chandrasekar et al 2004). Furthermore, the combination therapy of voriconazole plus caspofungin was more effective than monotherapy in a neutropenic guinea pig model of invasive aspergillosis (Kirkpatrick et al 2002).

\section{Clinical use against fungal pathogens Aspergillosis}

Invasive aspergillosis is a serious, life-threatening infection with a high attributable mortality rate $(65 \%-90 \%)$ in immunocompromised patients. Furthermore, the incidence of this infection has increased substantially over the past two decades. Voriconazole has been used as the primary therapy and as a salvage therapy in a number of studies of immunosuppressed patients with invasive aspergillosis, with encouraging results (Table 6). The largest of these studies, a randomized clinical trial, compared voriconazole with amphotericin B as the primary therapy in 277 hematological patients with invasive aspergillosis. At week 12, a successful outcome was observed in $53 \%$ of patients given voriconazole and $32 \%$ of those given amphotericin $\mathrm{B}$. The survival rate at 12 weeks showed a significant advantage for the voriconazole group with $71 \%$ survival compared with $58 \%$ in the amphotericin group (Herbrecht et al 2002).

In an open, noncomparative, multicenter study of immunocompromised patients with invasive aspergillosis, 
treatment with voriconazole was effective in $48 \%$ of cases (Denning et al 2002). In two large studies of patients refractory to or intolerant of treatments for fungal infections, efficacy rates of 44\% and 53\% (de Pauw 1999; Perfect et al 2003, respectively) were reported for aspergillosis treated with voriconazole.

In a recent, retrospective cohort study of patients with A. terreus infections, in which voriconazole was compared with other antifungal therapies, a decrease in mortality at 12 weeks was observed (55.8\% vs $73.4 \%$, respectively) in those patients who received voriconazole (Steinbach et al 2004).

One of the most important problems in the treatment of cerebral aspergillosis is the poor penetration of antifungal agents into the central nervous system (CNS). However, treatment with voriconazole results in measurable drug levels in the CSF, which may exceed the MIC for Aspergillus spp (Lutsar et al 2003). In a retrospective study, patients with CNS aspergillosis were treated with voriconazole as salvage therapy, for a median of 51 days. Complete and partial responses were observed in $35 \%$ of all patients, with a survival rate of $31 \%$ (Troke et al 2003).

Recently, a global response rate of $55 \%$ was also recorded in 20 patients with bone aspergillosis treated with voriconazole (18 as a salvage therapy and two as a primary therapy) (Mouas et al 2005). Similar results have been reported in other studies involving bone-marrow and solidorgan transplant recipients, critically ill patients, hematological malignancies, chronic granulomatous disease, and other immunocompromising conditions (Table 6) (Caillot et al 1997; Walsh, Lutsar, et al 2002; Alvarez-Lerma et al 2005; Diaz-Pedroche et al 2005).

Numerous case reports have also been published documenting the successful use of voriconazole in the treatment of invasive aspergillosis in hematological malignancies, both in pediatric patients (Cesaro et al 2003; Chow et al 2003; Shouldice et al 2003; Lassaletta et al 2004; Rosen-Wolff et al 2004) and adults ('t Hek et al 1998; Verweij et al 1999; Hwang et al 2001; Mattei et al 2002; Garbino et al 2003; Sambatakou et al 2003; Azzola et al 2004; Eibl et al 2004).

The efficacy of voriconazole in the treatment of Aspergillus spp infections has also been confirmed in a number of individual case reports, including infections of the skin (La Nasa et al 2004), bone (Swift and Denning 1998; Stratov et al 2003), mediastinum (Levin et al 2004), eye (Aliyeva et al 2004; Bethell et al 2004), prosthetic valve
(Reis et al 2005), and CNS (Moling et al 2002; de Lastours et al 2003; Marbello et al 2003; Tattevin et al 2004; Schwartz et al 2005). Furthermore, recently published data suggest that voriconazole is a useful adjunctive therapy for allergic bronchopulmonary aspergillosis in children with cystic fibrosis (Hilliard et al 2005).

According to these studies, voriconazole is likely to become the new standard therapy for invasive aspergillosis.

\section{Candidiasis}

Infections due to Candida spp are an important cause of morbidity and mortality in immunocompromised patients. Furthermore, Candida spp are the fourth most common source of nosocomial bloodstream infections in the USA. Clinical findings suggest that voriconazole may become an effective therapeutic option for candidiasis because of increases in fluconazole-susceptible or -resistant Candida isolates. For instance, voriconazole, alone or with caspofungin, was useful in the management of endogenous Candida endophthalmitis (Breit et al 2005). The successful treatment with voriconazole of keratitis, retinitis, or chorioretinitis due to Candida spp has also been reported (Granados et al 2004; Jang et al 2005; Varma et al 2005). There are several documented cases of a successful response to voriconazole in the treatment of infections caused by uncommon or fluconazole-resistant species (Corpus et al 2004; Fabry et al 2005): non-C. albicans peritonitis in peritoneal dialysis patients (Kleinpeter 2004), and Candida infection of the subacromial bursa (Khazzam et al 2005).

In a multicenter, randomized, double-blind, doubledummy study, voriconazole was at least as effective as fluconazole in the treatment of oral and esophageal candidiasis in immunocompromised patients (Ally et al 2001). Previously, voriconazole had been used successfully as a salvage therapy in fluconazole-refractory mucosal candidiasis in HIV-positive patients (Hegener et al 1998).

In systemic candidiasis, a $56 \%$ complete or partial cure rate was observed with voriconazole in patients intolerant of other antifungal agents or with refractory infections (Ostrosky-Zeichner, Oude Lashof, et al 2003). A similar global response rate $(55 \%)$ for invasive candidiasis was also reported in a large salvage therapy study that investigated the effectiveness of voriconazole against a wide range of invasive mycoses (Perfect et al 2003). Most recently, data from a large multicenter, randomized trial has been published that compares the efficacy of voriconazole with a sequential therapy 
comprising conventional amphotericin B followed by fluconazole in 422 nonneutropenic patients with candidemia (Kullberg et al 2005). At the primary end point (12 weeks after the end of therapy), voriconazole was not inferior to the amphotericin B/fluconazole regimen. In the secondary analysis, the success rate of voriconazole at the end of therapy was $65 \%$. Voriconazole cleared the bloodstream of Candida as rapidly as amphotericin B, followed by fluconazole, and with fewer toxic effects. The majority of infections were due to nonCandida albicans species (55\%) but there was a similar distribution of species in the two treatment groups. The successful response rates were similar between the voriconazole and amphotericin B/ fluconazole arms, except for $C$. tropicalis. The proportion of patients with C. tropicalis that responded satisfactorily was substantially higher in the voriconazole treatment group, although these isolates were also susceptible to amphotericin B.

The efficacy of voriconazole in the treatment of invasive candidiasis in pediatric patients has also been reported on a compassionate-use basis (Walsh, Lutsar, et al 2002) and as the primary therapy for a preterm infant with a disseminated fluconazole-resistant $C$. albicans infection (Muldrew et al 2005).

The broader activity spectrum of voriconazole, its greater safety than that of amphotericin B, and the availability of intravenous and oral formulations make voriconazole an important new treatment option for invasive Candida infections.

\section{Scedosporium/Pseudallescheria infections}

Scedosporium apiospermum and S. prolificans, which are generally amphotericin B resistant, have emerged as major pathogens among immunocompromised patients. The clinical experience of voriconazole treatment for scedosporiosis and pseudallescheriasis usually reflects the in vitro results, showing excellent activity against $S$. apiospermum but only modest activity against $S$. prolificans, organisms found mostly in Spain and Australia (CuencaEstrella et al 1999; Carrillo and Guarro 2001). The usefulness of voriconazole therapy in soft-tissue and subcutaneous infections of $S$. apiospermum has been reported in several immunocompromised patients (Montejo et al 2002; Bosma et al 2003; Schaenman et al 2005). Posttraumatic $S$. apiospermum keratitis or endophthalmitis have also been successfully treated with systemic and topical voriconazole (Fortun et al 2003; Nulens et al 2003; Figueroa et al 2004; Hernandez et al 2004). One of the rare cases of chronic vertebral osteomyelitis caused by $S$. apiospermum was treated with salvage surgery and long-term voriconazole therapy, leading to an adequate two-year outcome (German et al 2004).

The effectiveness of voriconazole in the treatment of $S$ apiospermum invasive pulmonary infections in children with hematological malignancies has also been reported (Jabado et al 1998; Walsh, Lutsar, et al 2002; Klopfenstein et al 2003). Recently, chronic meningitis caused by S. apiospermum was documented in a woman with no predisposing condition. She was treated with an entirely oral course of voriconazole for 12 months; two years after the cessation of therapy, the patient remained symptom-free (Danaher and Walter 2004). In recent years, several publications have reported successful outcomes when disseminated $S$. apiospermum infections were treated with voriconazole in patients with different underlying conditions: corticosteroid therapy (Munoz et al 2000), solid-organ transplant recipient (Fortun et al 2003; Ahmed et al 2004; Husain et al 2005), acute myeloid leukemia (Girmenia et al 1998), and failure or intolerance of standard antifungal therapies (Baden et al 2003).

The efficacy of voriconazole in the treatment of deep infections caused by Pseudallescheria boydii (sexual form of $S$. apiospermum) has also been reported: brain abscess (Nesky et al 2000; Castiglioni et al 2002), pulmonary empyema (Perlroth and Miller 2004), pneumonia (Chaney et al 2004), fungus ball (Garci et al 2003), osteomyelitis (Kanafani et al 2004), and chronic meningitis (Poza et al 2000).

S. prolificans is highly resistant to antifungal agents, both in vitro and in vivo. However, individual case reports have noted successful outcomes with voriconazole treatment for $S$. prolificans osteomyelitis and disseminated infections in combination with surgery (Studahl et al 2003), caspofungin (Steinbach et al 2003), or terbinafine (Gosbell et al 2003; Howden et al 2003).

\section{Fusarium infections}

Although Fusarium infections are known to cause major morbidity and mortality in immunocompromised hosts, the clinical data currently available on the efficacy of voriconazole in the treatment of these infections are promising. Severe hypopyon keratitis (Reis et al 2000) and a corneal abscess (Polizzi et al 2004), both caused 
by $F$. solani, that did not respond to common antifungal agents, underwent clinical cures with topical and systemic voriconazole therapy. The first two cases of Fusarium soft-tissue infection treated successfully with voriconazole were reported in 2004: a proven $F$. dimerum infection of the foot with a possible pulmonary infection 10 days after a sibling allogeneic stem-cell transplant (Bigley et al 2004), and a proven Fusarium spp nodular lesion on the left arm of a woman with Evans syndrome (Guimera-Martin-Neda et al 2004).

There are several documented cases of disseminated Fusarium infections that responded successfully when voriconazole was added to the therapy: a $F$. solani infection with skin and cerebellar lesions in a neutropenic patient (Vincent et al 2003); a disseminated F. oxysporum infection with skin localization in a woman with a relapse of B-acute leukemia during induction chemotherapy (Durand-Joly et al 2003); a disseminated Fusarium spp infection with skin manifestations in a severely neutropenic and immunocompromised host (Consigny et al 2003); a three-year-old girl with severe aplastic anemia and skin, chest wall, and splenic lesions caused by F. oxysporum (Rodriguez et al 2003); and a 10 -yearold neutropenic girl, treated for acute myeloid leukemia, with disseminated fusariosis uncontrolled by amphotericin B (Petit et al 2005). An immunocompromised child with $F$. solani endocarditis survived after treatment with voriconazole, even though the organism was resistant in vitro to all available antifungal agents (Guzman-Cottrill et al 2004). Two studies of salvage therapies with voriconazole for less common fungal infections reported satisfactory global responses in seven (50\%) of 14 patients (Baden et al 2003; Perfect et al 2003). This proportion is similar to that in pooled data presented to the FDA, wherein nine (43\%) of 21 patients with fusariosis had a complete or partial response to voriconazole provided on a com-passionate-use basis. In contrast, a recent publication reported a patient with acute leukemia who suffered breakthrough fusariosis while receiving voriconazole (Cudillo et al 2005). However, reports of the successful use of voriconazole therapy in patients with fusariosis and in vitro studies showing lower MIC values for voriconazole against Fusarium species than for itraconazole or amphotericin B suggest a potential role for voriconazole as a lifesaving therapy for immunocompromised hosts with Fusarium infections.

\section{Acremonium infections}

Acremonium spp are ubiquitous saprobes in the soil and an infrequent cause of infections in immunocompetent and immunocompromised hosts. Two fungemia episodes caused by Acremonium spp were successfully treated with voriconazole after the failure of amphotericin B therapy (Mattei et al 2003). Another successful outcome has been reported after oral voriconazole treatment for disseminated Acremonium infection associated with pacemaker-related endocarditis and endophthalmitis in a patient with no underlying condition (Heitmann et al 1997). Thus, voriconazole can be considered an effective salvage therapy for invasive Acremonium infections.

\section{Other organisms}

Voriconazole may also become a therapy option for less common fungal pathogens. Case reports have suggested that voriconazole is clinically efficacious in disseminated Coccidioides immitis infections. A patient with disseminated nonmeningeal coccidioidomycosis, clinically unresponsive to a prolonged course of amphotericin B, responded favorably to voriconazole monotherapy without adjunctive surgical intervention (Prabhu et al 2004). Another patient with coccidiodal meningitis responded to high-dose voriconazole therapy after the failure of fluconazole treatment (Cortez et al 2003).

Eleven HIV-infected patients from Thailand had disseminated Penicillium marneffei infections; eight of the nine assessable patients responded successfully to voriconazole (Schlamm and Supparatpnyo 2003). Moreover, the high response rate among patients with penicilliosis $(90 \%)$ and phaeohyphomycosis $(100 \%)$ to voriconazole when it is used as a salvage therapy suggests that this drug has a significant role in the management of refractory or less common fungal infections (Perfect et al 2003).

A case of disseminated Paecilomyces lilacinus, successfully treated with voriconazole, has been described in an AIDS patient. The strain isolated was susceptible in vitro to voriconazole (MIC, $0.12 \mathrm{mg} / \mathrm{L}$ ) but resistant to itraconazole (MIC, $2 \mathrm{mg} / \mathrm{L}$ ) and amphotericin B (MIC, $>8 \mathrm{mg} / \mathrm{L}$ ), demonstrating a good correlation between the in vivo and in vitro data (Martin et al 2002).

\section{Clinical use in patients at risk Hematological patients}

The role of voriconazole as an empirical treatment for patients with febrile neutropenia remains unresolved, and 
in fact, voriconazole is currently not licensed for this indication. The results of a large, international, multicenter, randomized study that compared voriconazole with liposomal amphotericin B for the empirical treatment of febrile neutropenic patients have been controversial (Walsh, Pappas, et al 2002). The analysis was performed on a modified intent-to-treat basis for a total of 837 enrolled febrile neutropenic patients $(72 \%$ with hematological malignancies), 415 of whom received voriconazole and 422 of whom received liposomal amphotericin B. According to the predefined end point of this study (noninferiority), voriconazole did not achieve this goal relative to liposomal amphotericin $\mathrm{B}$; the overall success rates were $26 \%$ in voriconazole-treated patients and $30.6 \%$ in liposomal amphotericin-B-treated patients. Complete or partial response rates of the 19 patients with baseline fungal infections favored liposomal amphotericin B (66.7\% vs $46.2 \%$ for voriconazole). However, breakthrough fungal infections occurred in eight patients $(1.9 \%)$ in the voriconazole group compared with 21 patients $(5 \%)$ in the liposomal amphotericin B group $(p=0.02)$. Moreover, patients receiving voriconazole in the stratified cohort with a high risk of fungal infection (those with allogeneic transplants or relapsed leukemia) demonstrated an even more pronounced reduction in invasive fungal infections than patients receiving liposomal amphotericin B (two of 143 [1.4\%] vs 13 of $141,[9.2 \%]$, respectively; $p=0.003$ ). Furthermore, the voriconazole group had fewer cases of severe infusion-related reactions $(\mathrm{p}<0.01)$ or nephrotoxicity $(\mathrm{p}<0.001)$, although the number of deaths was similar in both groups. Based on these results, voriconazole did not receive FDA approval as an empirical antifungal therapy, leading to the paradox that an agent licensed and considered to be the antifungal agent of choice for the treatment of documented Aspergillus infection was not deemed suitable for the basic objective of an empirical antifungal therapy (to prevent an invasive fungal infection or treat an occult invasive fungal infection that is not documented). This is attributable to one of the potential drawbacks in using defervescence as a criterion in the composite end point of success, because other causes of fever can confound the assessment of a true antifungal effect. Thus, on the basis of currently available evidence, voriconazole appears to be a suitable alternative to conventional liposomal amphotericin $B$ as an empirical antifungal therapy in patients with persistent fever and neutropenia (Klastersky 2004).

Once a diagnosis of invasive fungal infection is made, the use of voriconazole for the treatment of hematological patients is as recommended above, according to the causal organism. Another important issue in hematological patients is that any delay in the scheduled treatment of the underlying malignancy until complete resolution of a probable or proven fungal infection may have major implications for the prognosis. In this context, secondary prophylaxis with voriconazole was successful in 11 patients with acute leukemia with previous Aspergillus $(\mathrm{n}=10)$ or Candida $(\mathrm{n}=1)$ infections. Nine patients were scheduled for allogeneic stem-cell transplantation, and two for consolidation therapy for acute leukemia. They received voriconazole $(400 \mathrm{mg} /$ day) intravenously or orally for 44-245 days. None of the patients had a relapse of the fungal infection, and the scheduled treatments for leukemia were delayed only once (Cordonnier et al 2004). This small but homogeneous series indicates that voriconazole may be useful in preventing fungal relapse in leukemic patients during at-risk periods. However, caution is required when prescribing voriconazole to hematopoietic stem-cell transplant recipients. It is noteworthy that several antineoplastic agents commonly used in the conditioning regimen are metabolized by the CYP3A4 enzyme system. If a patient receiving voriconazole for a preexisting fungal infection is scheduled to undergo a hematopoietic stem-cell transplant, voriconazole should be stopped at least 30 hours prior to the preparative regimen, and restarted at least 48 hours after conditioning chemotherapy (usually on day +1 ). Of course, non-azole antifungals can be administered during the preparative regimen. The main clinically significant drug interactions with voriconazole are summarized in Table 3 .

\section{Critically ill patients}

Approximately $10.4 \%$ of the infectious episodes in an Intensive Care Unit (ICU) are related to a Candida spp infection, most of which are hospital-acquired (Alberti et al 2002). This rate may be an underestimation because at least $4 \%$ of deaths in ICUs present with an unexpected fungal infection on postmortem examination (Dimopoulos et al 2004). Furthermore, ICU admission itself has become an independent risk factor for Candida infection (Puzniak et al 2004; Tortorano et al 2004), and has also been associated with significant mortality among critically ill patients (Kalenic et al 2001).

The choice of the best first-line treatment for candidemia remains controversial, especially in critically ill patients. Successful therapy with compassionate use of voriconazole for the treatment of candidemia and invasive candidiasis in 
patients intolerant of or refractory to other antifungal agents has been reported (Ostrosky-Zeichner, Oude Lashof, et al 2003). This study showed that voriconazole may be a suitable agent for salvage treatment of invasive candidiasis, even in the setting of previous azole exposure and $C$. krusei infection. These findings have also been confirmed by two Spanish studies (Munoz et al 2005; Pemán et al 2005).

A Spanish observational multicenter study (AlvarezLerma et al 2005) assessed the clinical use and tolerability of voriconazole for the treatment of fungal infections in critically ill patients. The prescription of voriconazole was based on the presence of an episode of fungal infection, and identification of the genus and species of the causative pathogen, which had previously been treated with other antifungal drugs. Voriconazole was effective in $50 \%$ of patients. The drug was well tolerated and treatment discontinuation because of adverse events was not necessary.

Recently, the first randomized, prospective, multicenter study of nonneutropenic patients with candidemia treated with voriconazole or amphotericin B deoxycholate has shown equivalent results for the efficacy and mortality in both treatment arms (Kullberg et al 2005). The incidence of renal dysfunction was significantly lower in the voriconazole group. Although the incidence of visual disturbances was slightly higher in this group, these side effects were usually transient and resolved after the patient had become tolerant of the drug or the drug had been discontinued. About half the patients included in this study were admitted to an ICU. The only limitation to the intravenous administration of voriconazole in critically ill patients could be the accumulation and toxicity of its excipient (cyclodextrin) in patients with severe renal dysfunction. Data for patients undergoing renal replacement therapy are lacking.

The efficacy and safety of voriconazole has also been demonstrated in the treatment of invasive aspergillosis and other less common mould infections (Herbrecht et al 2002; Perfect et al 2003). These results provide sufficient evidence to justify the use of voriconazole as a first-line therapy, alone or in combination, in suspected or proven invasive aspergillosis or other mould infections in ICU settings.

The encouraging clinical experience with this drug suggests that voriconazole is a new therapeutic alternative in critically ill patients, not only as a salvage treatment, but also as a first-line option in suspected or proven Candida and Aspergillus infections.

\section{Other patients at risk}

\section{Solid-organ transplantation}

Before voriconazole came into use, mortality due to invasive mould infections in solid-organ transplant (SOT) recipients was very high, despite therapy with amphotericin B. Preliminary results for voriconazole administered on a compassionate-use basis in patients with these infections has been promising. Voriconazole was administered for an average of 80 days to four patients with invasive $A$. fumigatus infection and to another patient with invasive ocular S. apiospermum infection. All of these patients had been treated previously with a lipid formulation of amphotericin B, and cultures were persistently positive despite a cumulative dose of $3 \mathrm{~g}$ (Fortun et al 2003). A clinical response to voriconazole was observed in all patients (three with a complete response; a partial response in two), and a microbiological response was observed in all but one patient. Furthermore, a good correlation between the MIC of voriconazole and outcome was observed. However, an increase in the levels of immunosuppressive drugs (tacrolimus or cyclosporine) was detected in all patients.

In another study (Baden et al 2003) in which voriconazole was used in a compassionate release program, 35 (78\%) of patients had invasive Aspergillus, three (7\%) had Fusarium infections, and two (4\%) had Scedosporium infections. Overall response rates were nine (20\%) complete responses, 17 (38\%) partial responses, 15 (33\%) failures, and four (9\%) patients were intolerant. Seven of the eight $(88 \%)$ patients with sinus or CNS disease exhibited stabilization of the invasive fungal infection. In a total of 9128 days of therapy, only four serious adverse events that were considered possibly or probably drug-related were observed in two patients.

Therefore, voriconazole may be considered an effective and safe therapy for the treatment of invasive mould infections in SOT recipients. However, to avoid toxicity with this drug, the doses of immunosuppressive drugs must be reduced (Table 3 ).

\section{HIV Infection or AIDS}

A multicenter, randomized, double-blind, double-dummy study compared voriconazole with fluconazole for the treatment of esophageal candidiasis in 391 immunocompromised patients, most of whom had AIDS 
(Ally et al 2001). There was no difference between the two groups with respect to cure, as determined by esophagoscopy (98.3\% of patients who received voriconazole and $95.1 \%$ of patients who received fluconazole achieved cure). Another small open-label, noncomparative study evaluated the efficacy of voriconazole treatment for fluconazolerefractory esophageal candidiasis in 12 patients with AIDS. At day 7, six patients were cured, and the conditions of three showed marked improvement; one other patient was cured after two weeks of therapy, and there was no response in two patients (Hegener et al 1998).

Voriconazole demonstrates excellent in vitro activity against $C$. neoformans and achieves good CSF levels, but no clinical trial results and only a few case reports of voriconazole used against cryptococcal meningitis have been published. In a case report of relapsing meningitis due to a fluconazole-resistant $C$. neoformans in a patient with advanced HIV infection, maintenance therapy with voriconazole was unsuccessful in preventing a recurrence of meningitis (Friese et al 2001). In another study that focused on patients who were refractory to or intolerant of treatment for fungal infections, the response rate for cryptococcosis was 39\% (Perfect et al 2003).

Thus, although voriconazole cannot be recommended presently to treat HIV patients with disseminated or meningeal cryptococcosis, its appropriate in vitro activity against $C$. neoformans, suitable distribution in CSF and the results obtained in the before mentioned studies make voriconazole a promising drug in this setting. However, there is need for more clinical and pharmacological studies in HIV patients to assess pharmacokinetic effects, with or without clinical relevance, derived from the coadministration with protease inhibitors or other antiretroviral drugs (efavirenz).

\section{Pediatric patients}

Children require higher doses of voriconazole than adults to attain similar serum concentrations over time. Based on limited pharmacokinetic analyses, it seems that a pediatric dosage of $11 \mathrm{mg} / \mathrm{kg}$ administered every 12 hours is approximately bioequivalent to an adult dose of $4 \mathrm{mg} / \mathrm{kg}$ given every 12 hours (Steinbach 2005). The largest pediatric report of voriconazole treatment was an open-label compassionate-use study of 69 children between the ages of nine months and 15 years for the treatment of an invasive fungal infection (Walsh, Lutsar, et al 2002). The most common underlying condition was hematological malignancy, and the most frequent fungal pathogens were Aspergillus spp (72\%) and Scedosporium spp (14\%). After a mean period of voriconazole therapy of three months, 26 patients $(45 \%)$ had a complete or partial response, four patients had a stable response, and 25 failed therapy. Stratifying the outcomes by pathogen revealed a complete or partial response in $43 \%$ of patients with aspergillosis, $50 \%$ of patients with candidemia, and $63 \%$ of patients with scedosporiosis. Only three patients were withdrawn from the study because of toxicity. Common adverse effects included elevation in transaminases or bilirubin, rash, abnormal vision, and photosensitivity. The median plasma concentration of voriconazole was $1566 \mathrm{ng} / \mathrm{mL}$ for children receiving $\geq 4 \mathrm{mg} / \mathrm{kg}$ intravenously bid, which is lower than the median plasma concentrations $(5671 \mathrm{ng} / \mathrm{mL})$ in a control population of adults receiving $4 \mathrm{mg} / \mathrm{kg}$ bid. Another study reported the experiences of seven children with oncohematological malignancies and invasive aspergillosis treated with voriconazole. Responses were complete in two patients, partial in two patients, and stable in one; and there was no response in the two other patients (Cesaro et al 2003).

Recently, treatment with voriconazole was reported in eight pediatric patients with invasive bone aspergillosis, who were mainly affected by chronic granulomatous disease, in a worldwide series of 20 cases. At the end of therapy, four $(50 \%)$ patients had a satisfactory response (one complete and three partial) to voriconazole (Mouas et al 2005).

These data lend support to the use of voriconazole in the pediatric population as an alternative treatment for invasive mycosis unresponsive to conventional antifungal agents. However, additional clinical trials, including kinetic studies, are required to further clarify the role of voriconazole in the treatment of these infections in children.

\section{Voriconazole in combination therapies}

The availability of new antifungal agents with single mechanisms of action and improved tolerability has widened the possibilities for the use of combination antifungal therapies for difficult-to-treat opportunistic mycoses. Few randomized clinical trials have examined the role of this type of therapy for invasive mycoses, and no prospective randomized trial of antifungal combinations has been completed for invasive mould infections. The results of in vitro studies and those with animal models suggest that combination therapies with azoles and echinocandins may 
have additive activity against Aspergillus species (Kirkpatrick et al 2002; Perea et al 2002).

A recent study evaluated the outcomes of hematological patients with pulmonary aspergillosis, who received either voriconazole or a combination of voriconazole and caspofungin as a salvage therapy. The combination therapy was associated with an improved three-month survival rate and with reduced mortality, compared with the voriconazole monotherapy (Marr et al 2004). Apart from salvage therapy results, further studies are required to show that a combination therapy with voriconazole is better than a single-drug treatment as the first-line therapy for invasive aspergillosis in selected patients.

A growing number of case reports of combination therapies for rare and emerging fungal infections (eg, Scedosporium spp, Fusarium spp, Cladophialophora bantiana) have described the beneficial action when voriconazole was combined with either amphotericin B, caspofungin, or terbinafine (Durand-Joly et al 2003; Howden et al 2003; Steinbach et al 2003; Trinh et al 2003; Cantón et al 2005). Combination therapies with voriconazole may have value in select populations of patients at high risk of death due to candidiasis, trichosponorosis, or blastoschizomycosis, who are undergoing azole-based prophylaxis, and high-risk patients with multiple-species candidemia (Kontoyiannis and Lewis 2004). However, the possible benefits of combination therapies with voriconazole for disseminated cryptococcosis and invasive candidiasis (or other emerging yeasts) must be clarified. At present, the combination of amphotericin B plus flucytosine and monotherapies with either fluconazole or caspofungin may be more desirable in these contexts.

Most results and reports indicate that a combination regimen may be an effective option in salvage therapies for invasive aspergillosis and probably other difficult-to-treat invasive fungal infections. Furthermore, the additive in vitro interactions of voriconazole and echinocandins suggest a great potential for such a combination therapy and confirm the need for further investigations.

\section{Ecological impact of voriconazole use}

Infections with voriconazole-resistant fungi may become problematic, because organisms with decreased susceptibility have been noted. Breakthrough fungal infections occurred in 13 of 139 patients ( $9 \%$ ) who received voriconazole at the Fred Hutchinson Cancer Research Center in Seattle, USA, during a five-year period. Candida glabrata fungemia occurred in four patients and zygomycetes were found in six patients (Imhof et al 2004). In a prospective surveillance of 27 patients with zygomycosis at the M.D. Anderson Cancer Center, nearly all patients either had leukemia $(n=14)$ or were allogeneic bone-marrow transplant recipients $(\mathrm{n}=13)$. All the zygomycetes isolates $(74 \%$ of which were of the genus Rhizopus) were voriconazoleresistant. Previous exposure to voriconazole was identified by multivariate analysis as a significant risk factor for zygomycosis (Kontoyiannis et al 2005). The association between the use of voriconazole and zygomycosis is further supported by several reports of breakthrough zygomycosis in patients receiving voriconazole (Blin et al 2004; Kobayashi et al 2004; Marty et al 2004; Mattner et al 2004; Siwek et al 2004; Oren 2005; Vigouroux et al 2005). Considering the growing number of reports, this issue requires further attention. However, the definite causal relationship between treatment with voriconazole and the emergence of breakthrough infections caused by zygomycetes must still be established in larger case series. Although the use of voriconazole may have contributed to the occurrence of zygomycosis, there are some data indicating that the incidence of zygomycosis was increasing before the introduction of voriconazole to clinical practice (Kauffman 2004). It is possible that the control of a highly aggressive infection such as invasive aspergillosis permits patients to live long enough to develop a subsequent breakthrough infection with persistent immunosuppression. For instance, the first case of disseminated infection with Prototheca zopfii was reported following an unrelated stemcell transplantation for leukemia, during the long-term administration of voriconazole for the treatment of pulmonary aspergillosis (Lass-Florl et al 2004).

\section{Conclusions}

Voriconazole is a new triazole with high oral bioavailability, large volume of distribution (with excellent CNS penetration), and elimination through hepatic metabolism by cytochrome $\mathrm{P} 450$ isozymes.

The side-effect profile of voriconazole presents an acceptable safety and tolerability spectrum. Transient visual disturbances, liver enzyme abnormalities, and skin rashes are the most frequently reported, but rarely lead to discontinuation of treatment. The potential for drug-drug interactions is high, because of the extensive hepatic metabolism of voriconazole. Therefore, careful attention to dosage is required, and its serum levels and the effects of 
any interacting drug should be monitored. In young children, it may be necessary to increase the dose administered because of the rapid metabolism of the drug in this population. Consistent with the high oral bioavailability, an early switch from intravenous to oral therapy makes this drug attractive for prolonged oral tratment after discharge from the hospital.

Voriconazole has shown excellent in vitro activity against a wide range of yeasts (including fluconazoleresistant species and $C$. neoformans), dimorphic fungi, opportunistic moulds (Aspergillus spp., Fusarium spp., and $S$. apiospermum), and the amphotericin-B-resistant $A$. fumigatus and $A$. terreus isolates. However, it lacks in vitro activity against zygomycetes.

According to the in vitro and in vivo data reviewed, voriconazole can be considered as the new standard treatment for invasive aspergilliosis, either for primary or salvage therapy. Furthermore, voriconazole displays clinical efficacy in patients with fluconazole-resistant and fluconazole-susceptible Candida invasive infections, bone and CNS aspergillosis, and various refractory fungal infections.

Additive in vitro interactions and good clinical response in patients with invasive fungal infections treated with a combination of voriconazole and echinocandins, amphotericin B or terbinafine suggest a great potential for such a combination therapy which needs to be confirmed in further investigations.

\section{References}

't Hek LG, Verweij PE, Weemaes CM, et al. 1998. Successful treatment with voriconazole of invasive aspergillosis in chronic granulomatous disease. Am J Respir Crit Care Med, 157:1694-6.

Ahmed J, Ditmars DM, Sheppard T, et al. 2004. Recurrence of Scedosporium apiospermum infection following renal retransplantation. Am J Transplant, 4:1720-4.

Alberti C, Brun-Buisson C, Burchardi H, et al. 2002. Epidemiology of sepsis and infection in ICU patients from an international multicentre cohort study. Intensive Care Med, 28:108-21.

Aliyeva SE, Ullmann AJ, Kottler UB, et al. 2004. Histological examination of an eye with endogenous Aspergillus endophthalmitis treated with oral voriconazole: a case report. Graefes Arch Clin Exp Ophthalmol, 242:887-91.

Ally R, Schurmann D, Kreisel W, et al. 2001. A randomized, double-blind, double-dummy, multicenter trial of voriconazole and fluconazole in the treatment of esophageal candidiasis in immunocompromised patients. Clin Infect Dis, 33:1447-54.

Alvarez-Lerma F, Nicolas-Arfelis J, Rodriguez-Borregan J, et al. 2005. Clinical use and tolerability of voriconazole in the treatment of fungal infections in critically ill patients. $J$ Chemother, 17: 417-27.

Azzola A, Passweg JR, Habicht JM, et al. 2004. Use of lung resection and voriconazole for successful treatment of invasive pulmonary Aspergillus ustus infection. J Clin Microbiol, 42:4805-8.
Baden LR, Katz JT, Fishman JA, et al. 2003. Salvage therapy with voriconazole for invasive fungal infections in patients failing or intolerant to standard antifungal therapy. Transplantation, 76:16327.

Barchiesi F, Spreghini E, Maracci M, et al. 2004. In vitro activities of voriconazole in combination with three other antifungal agents against Candida glabrata. Antimicrob Agents Chemother, 48:3317-22.

Bethell D, Hall G, Goodman TR, et al. 2004. Resolution of orbitocerebral aspergillosis during combination treatment with voriconazole and amphotericin plus adjunctive cytokine therapy. $J$ Pediatr Hematol Oncol, 26:304-7.

Bigley VH, Duarte RF, Gosling RD, et al. 2004. Fusarium dimerum infection in a stem cell transplant recipient treated successfully with voriconazole. Bone Marrow Transplant, 34:815-17.

Blin N, Morineau N, Gaillard F, et al. 2004. Disseminated mucormycosis associated with invasive pulmonary aspergillosis in a patient treated for post-transplant high-grade non-Hodgkin's lymphoma. Leuk Lymphoma, 45:2161-3.

Bosma F, Voss A, van Hamersvelt HW, et al. 2003. Two cases of subcutaneous Scedosporium apiospermum infection treated with voriconazole. Clin Microbiol Infect, 9:750-3.

Boucher HW, Groll AH, Chiou CC, et al. 2004. Newer systemic antifungal agents: pharmacokinetics, safety and efficacy. Drugs, 64:1997-2020.

Breit SM, Hariprasad SM, Mieler WF, et al. 2005. Management of endogenous fungal endophthalmitis with voriconazole and caspofungin. Am J Ophthalmol, 139:135-40.

Burn AK, Fothergill AW, Kirkpatrick WR, et al. 2004. Comparison of antifungal susceptibilities to fluconazole and voriconazole of oral Candida glabrata isolates from head and neck radiation patients. $J$ Clin Microbiol, 42:5846-8.

Caillot D, Casasnovas O, Bernard A, et al. 1997. Improved management of invasive pulmonary aspergillosis in neutropenic patients using early thoracic computed tomographic scan and surgery. J Clin Oncol, 15:139-47.

Cantón E, Pemán J, Gobernado, M, et al. 2005. Synergistic activities of fluconazole and voriconazole with terbinafine against four Candida species determined by checkerboard, time-kill, and Etest methods. Antimicrob Agents Chemother, 49:1593-6.

Capilla J, Guarro J. 2004. Correlation between in vitro susceptibility of Scedosporium apiospermum to voriconazole and in vivo outcome of scedosporiosis in guinea pigs. Antimicrob Agents Chemother, 48:4009-11.

Capilla J, Serena C, Pastor FJ, et al. 2003. Efficacy of voriconazole in treatment of systemic scedosporiosis in neutropenic mice. Antimicrob Agents Chemother, 47:3976-8.

Carrillo AJ, Guarro J. 2001. In vitro activities of four novel triazoles against Scedosporium spp. Antimicrob Agents Chemother, 45:2151-3.

Castiglioni B, Sutton DA, Rinaldimg, et al. 2002. Pseudallescheria boydii (Anamorph Scedosporium apiospermum). Infection in solid organ transplant recipients in a tertiary medical center and review of the literature. Medicine (Baltimore), 81:333-48.

Cesaro S, Strugo L, Alaggio R, et al. 2003. Voriconazole for invasive aspergillosis in oncohematological patients: a single-center pediatric experience. Support Care Cancer, 11:722-7.

Chandenier J, Adou-Bryn KD, Douchet C, et al. 2004. In vitro activity of amphotericin B, fluconazole and voriconazole against 162 Cryptococcus neoformans isolates from Africa and Cambodia. Eur J Clin Microbiol Infect Dis, 23:506-8.

Chandrasekar PH, Cutright J, Manavathu E. 2000. Efficacy of voriconazole against invasive pulmonary aspergillosis in a guinea-pig model. $J$ Antimicrob Chemother, 45:673-6.

Chandrasekar PH, Cutright JL, Manavathu EK. 2004. Efficacy of voriconazole plus amphotericin B or micafungin in a guinea-pig model of invasive pulmonary aspergillosis. Clin Microbiol Infect, 10:925-8.

Chandrasekar PH, Manavathu E. 2001. Voriconazole: A second-generation triazole. Drugs Today (Barc), 37:135-48. 
Chaney S, Gopalan R, Berggren RE. 2004. Pulmonary Pseudallescheria boydii infection with cutaneous zygomycosis after near drowning. South Med J, 97:683-7.

Chavez M, Bernal S, Valverde A, et al. 1999. In-vitro activity of voriconazole (UK-109,496), LY303366 and other antifungal agents against oral Candida spp. isolates from HIV-infected patients. $J$ Antimicrob Chemother, 44:697-700.

Chow E, Moore T, Nielsen K. 2003. Case report: Invasive aspergillosis successfully treated with voriconazole without recurrence during subsequent bone marrow transplantation. Pediatr Infect Dis $J$, 22:1109-10

Chryssanthou E, Cuenca-Estrella M. 2002. Comparison of the Antifungal Susceptibility Testing Subcommittee of the European Committee on Antibiotic Susceptibility Testing proposed standard and the E-test with the NCCLS broth microdilution method for voriconazole and caspofungin susceptibility testing of yeast species. J Clin Microbiol, 40:3841-4.

Clancy CJ, Nguyen MH. 1998. In vitro efficacy and fungicidal activity of voriconazole against Aspergillus and Fusarium species. Eur J Clin Microbiol Infect Dis, 17:573-5.

Consigny S, Dhedin N, Datry A, et al. 2003. Successsful voriconazole treatment of disseminated fusarium infection in an immunocompromised patient. Clin Infect Dis, 37:311-13.

Cordonnier C, Maury S, Pautas C, et al. 2004. Secondary antifungal prophylaxis with voriconazole to adhere to scheduled treatment in leukemic patients and stem cell transplant recipients. Bone Marrow Transplant, 33:943-8.

Corpus K, Hegeman-Dingle R, Bajjoka I. 2004. Candida kefyr, an uncommon but emerging fungal pathogen: report of two cases. Pharmacotherapy, 24:1084-88.

Cortez KJ, Walsh TJ, Bennett JE. 2003. Successful treatment of coccidioidal meningitis with voriconazole. Clin Infect Dis, 36:161922.

Cudillo L, Girmenia C, Santilli S, et al. 2005. Breakthrough fusariosis in a patient with acute lymphoblastic leukemia receiving voriconazole prophylaxis. Clin Infect Dis, 40:1212-13.

Cuenca-Estrella M, Gomez-Lopez A, Mellado E, et al. 2004. In vitro activities of ravuconazole and four other antifungal agents against fluconazole-resistant or -susceptible clinical yeast isolates. Antimicrob Agents Chemother, 48:3107-11.

Cuenca-Estrella M, Rodriguez D, Almirante B, et al. 2005. In vitro susceptibilities of bloodstream isolates of Candida species to six antifungal agents: results from a population-based active surveillance programme, Barcelona, Spain, 2002-2003. J Antimicrob Chemother, 55:194-9.

Cuenca-Estrella M, Rodriguez-Tudela JL, Mellado E, et al. 1998. Comparison of the in-vitro activity of voriconazole (UK-109,496), itraconazole and amphotericin B against clinical isolates of Aspergillus fumigatus. J Antimicrob Chemother, 42:531-3.

Cuenca-Estrella M, Ruiz-Diez B, Martinez-Suarez JV, et al. 1999. Comparative in-vitro activity of voriconazole (UK-109,496) and six other antifungal agents against clinical isolates of Scedosporium prolificans and Scedosporium apiospermum.J Antimicrob Chemother, 43:149-51.

Danaher PJ, Walter EA. 2004. Successful treatment of chronic meningitis caused by Scedosporium apiospermum with oral voriconazole. Mayo Clin Proc, 79:707-8.

Dannaoui E, Meletiadis J, Mouton JW, et al. 2003. In vitro susceptibilities of zygomycetes to conventional and new antifungals. $J$ Antimicrob Chemother, 51:45-52.

de Lastours V, Lefort A, Zappa M, et al. 2003. Two cases of cerebral aspergillosis successfully treated with voriconazole. Eur J Clin Microbiol Infect Dis, 22:297-9.

de Pauw B. 1999. Clinical potential and experience of voriconazole [abstract]. Clin Microbiol Infect, 5(Suppl 3):7.
Denning D, Del Favero A, Gluckman E, et al. 1995. UK-109,496, a novel wide spectrum triazole derivative for the treatment of fungal infections: clinical efficacy in acute invasive aspergillosis [abstract]. 35th Interscience Conference on Antimicrobial Agents and Chemotherapy. September 17-20, San Francisco CA, USA. F80.

Denning DW, Ribaud P, Milpied N, et al. 2002. Efficacy and safety of voriconazole in the treatment of acute invasive aspergillosis. Clin Infect Dis, 34:563-71.

Diaz-Pedroche C, Cisneros J, Lumbreras C, et al. 2005. Treatment of invasive fungal infections with voriconazole. Evaluation of experience with compassionate use of voriconazole in Spain. Rev Esp Quimioter, 18:149-58

Diekema DJ, Messer SA, Hollis RJ, et al. 2003. Activities of caspofungin, itraconazole, posaconazole, ravuconazole, voriconazole, and amphotericin B against 448 recent clinical isolates of filamentous fungi. J Clin Microbiol, 41:3623-6.

Diekema DJ, Petroelje B, Messer SA, et al. 2005. Activities of available and investigational antifungal agents against Rhodotorula species. $J$ Clin Microbiol, 43:476-8.

Dimopoulos G, Piagnerelli M, Berre J, et al. 2004. Post mortem examination in the intensive care unit: still useful? Intensive Care Med, 30:2080-5.

Donnelly JP, de Pauw BE. 2004. Voriconazole - a new therapeutic agent with an extended spectrum of antifungal activity. Clin Microbiol Infect, 10(Suppl 1):107-17.

Drago M, Scaltrito MM, Morace G. 2004. In vitro activity of voriconazole and other antifungal agents against clinical isolates of Candida glabrata and Candida krusei. Eur J Clin Microbiol Infect Dis, 23:61924.

Dupont B, Denning D, Lode H, et al. 1995. UK-109,496, a novel widespectrum triazole derivative for the treatment of fungal infections: clinical efficacy in chronic invasive aspergillosis [abstract]. 35th Interscience Conference on Antimicrobial Agents and Chemotherapy. September 17-20, San Francisco CA, USA. F81.

Durand-Joly I, Alfandari S, Benchikh Z, et al. 2003. Successful outcome of disseminated Fusarium infection with skin localization treated with voriconazole and amphotericin B-lipid complex in a patient with acute leukemia. J Clin Microbiol, 41:4898-900.

Eibl M, Auner HW, Zinke-Cerwenka W, et al. 2004. High-risk AML complicated by pulmonary aspergillosis: successful treatment with nonmyeloablative stem cell transplantation and long-term administration of voriconazole. Ann Hematol, 83:133-6.

EMEA. 2002. Vfend: European Public Assessment Report [online]. Accessed 25 August 2005. URL: http://www.emea.eu.int/humandocs/ Humans/EPAR/vfend/vfend.htm.

EMEA. 2005. Committee for medicinal products for human use: European Public Assessment Report (EPAR): Vfend [online]. Accessed 25 August 2005. URL: http://www.emea.eu.int/humandocs/PDFs/EPAR/ vfend/404901en1.pdf.

Ernst EJ, Yodoi K, Roling EE, et al. 2002. Rates and extents of antifungal activities of amphotericin B, flucytosine, fluconazole, and voriconazole against Candida lusitaniae determined by microdilution, Etest, and time-kill methods. Antimicrob Agents Chemother, 46:57881.

Espinel-Ingroff A. 1998. In vitro activity of the new triazole voriconazole (UK-109,496) against opportunistic filamentous and dimorphic fungi and common and emerging yeast pathogens. J Clin Microbiol, 36:198202

Espinel-Ingroff A, Barchiesi F, Cuenca-Estrella M, et al. 2005. International and multicenter comparison of EUCAST and CLSI M27-A2 broth microdilution methods for testing susceptibilities of Candida spp. to fluconazole, itraconazole, posaconazole, and voriconazole. J Clin Microbiol, 43:3884-9.

Espinel-Ingroff A, Boyle K, Sheehan DJ. 2001. In vitro antifungal activities of voriconazole and reference agents as determined by NCCLS methods: review of the literature. Mycopathologia, 150:101-15. 
Espinel-Ingroff A, Fothergill A, Peter J, et al. 2002. Testing conditions for determination of minimum fungicidal concentrations of new and established antifungal agents for Aspergillus spp.: NCCLS collaborative study. J Clin Microbiol, 40:3204-8.

Espinel-Ingroff A, Pfaller M, Messer SA, et al. 2004. Multicenter comparison of the Sensititre YeastOne colorimetric antifungal panel with the NCCLS M27-A2 reference method for testing new antifungal agents against clinical isolates of Candida spp. $J$ Clin Microbiol, 42:718-21.

Espinel-Ingroff A, Rezusta A. 2002. E-test method for testing susceptibilities of Aspergillus spp. to the new triazoles voriconazole and posaconazole and to established antifungal agents: comparison with NCCLS broth microdilution method. J Clin Microbiol, 40:2101-7.

Fabry K, Verheyden F, Nelen G. 2005. Infection of a total knee prosthesis by Candida glabrata: a case report. Acta Orthop Belg, 71:119-21.

Favel A, Michel-Nguyen A, Datry A, et al. 2004. Susceptibility of clinical isolates of Candida lusitaniae to five systemic antifungal agents. $J$ Antimicrob Chemother, 53:526-9.

FDA. 2001. Determination of regulatory review period for purposes of patent extension; Vfend [online]. Accessed 25 August 2005. URL: http://www.fda.gov/ohrms/dockets/98fr/03-9578.pdf.

Figueroa MS, Fortun J, Clement A, et al. 2004. Endogenous endophthalmitis caused by Scedosporium apiospermum treated with voriconazole. Retina, 24:319-20.

Fortun J, Martin-Davila P, Sanchez MA, et al. 2003. Voriconazole in the treatment of invasive mold infections in transplant recipients. Eur $J$ Clin Microbiol Infect Dis, 22:408-13.

Friese G, Discher T, Fussle R, et al. 2001. Development of azole resistance during fluconazole maintenance therapy for AIDS-associated cryptococcal disease. AIDS, 15:2344-5.

Garau M, Pereiro M Jr, del Palacio A. 2003. In vitro susceptibilities of Malassezia species to a new triazole, albaconazole (UR-9825), and other antifungal compounds. Antimicrob Agents Chemother, 47:23424.

Garbino J, Rohner P, Kolarova L, et al. 2003. Successful treatment of pulmonary invasive aspergillosis with voriconazole in patients who failed conventional therapy. Infection, 31:241-3.

Garci J, Perkins A, Garau M, et al. 2003. Successful treatment with voriconazol of a Pseudallescheria boydii fungus ball in an HIVpositive patient with previous tuberculosis. Rev Iberoam Micol, 20:64 7.

George D, Miniter P, Andriole VT. 1996. Efficacy of UK-109496, a new azole antifungal agent, in an experimental model of invasive aspergillosis. Antimicrob Agents Chemother, 40:86-91.

German JW, Kellie SM, Pai MP, et al. 2004. Treatment of a chronic Scedosporium apiospermum vertebral osteomyelitis. Case report. Neurosurg Focus, 17:E9.

Ghannoum MA, Isham M, Sheehan DJ. 2005. Can we accurately assess antifungal cidality in vitro? Cidality studies of voriconazole and caspofungin against Candida species. Clin Microbiol Infect, 11(Suppl 2):573-4.

Ghannoum MA, Okogbule-Wonodi I, Bhat N, et al. 1999. Antifungal activity of voriconazole (UK-109,496), fluconazole and amphotericin $\mathrm{B}$ against hematogenous Candida krusei infection in neutropenic guinea pig model. J Chemother, 11:34-9.

Gil-Lamaignere C, Hess R, Salvenmoser S, et al. 2005. Effect of media composition and in vitro activity of posaconazole, caspofungin and voriconazole against zygomycetes. J Antimicrob Chemother, 55:101619.

Girmenia C, Luzi G, Monaco M, et al. 1998. Use of voriconazole in treatment of Scedosporium apiospermum infection: case report. J Clin Microbiol, 36:1436-8.
Girmenia C, Pizzarelli G, D’Antonio D, et al. 2003. In vitro susceptibility testing of Geotrichum capitatum: comparison of the E-test, disk diffusion, and Sensititre colorimetric methods with the NCCLS M27A2 broth microdilution reference method. Antimicrob Agents Chemother, 47:3985-8.

Girmenia C, Tuccinardi C, Santilli S, et al. 2000. In vitro activity of fluconazole and voriconazole against isolates of Candida albicans from patients with haematological malignancies. J Antimicrob Chemother, 46:479-83.

Gonzalez GM, Sutton DA, Thompson E, et al. 2001. In vitro activities of approved and investigational antifungal agents against 44 clinical isolates of basidiomycetous fungi. Antimicrob Agents Chemother, 45:633-5.

Gosbell IB, Toumasatos V, Yong J, et al. 2003. Cure of orthopaedic infection with Scedosporium prolificans, using voriconazole plus terbinafine, without the need for radical surgery. Mycoses, 46:2336.

Granados JM, Puerto N, Carrilero MJ. 2004. Efficiency of voriconazole in fungal keratitis caused by Candida albicans. Arch Soc Esp Oftalmol, 79:565-8.

Green B, Lawrence C, Marr K. 2000. Voriconazole for salvage therapy of fungal infection in patients with hematological malignancies and stem cell transplantation [abstract]. Program and Abstracts of the 38th Annual Meeting of the Infection Diseases Society of America. September 7-10, New Orleans, LA, USA. 41.

Groll AH, Kolve H, Ehlert K, et al. 2004. Pharmacokinetic interaction between voriconazole and cyclosporin A following allogeneic bone marrow transplantation. J Antimicrob Chemother, 53:113-14.

Guimera-Martin-Neda F, Garcia-Bustinduy M, Noda-Cabrera A, et al. 2004. Cutaneous infection by Fusarium: successful treatment with oral voriconazole. Br J Dermatol, 150:777-8.

Guzman-Cottrill JA, Zheng X, Chadwick EG. 2004. Fusarium solani endocarditis successfully treated with liposomal amphotericin B and voriconazole. Pediatr Infect Dis J, 23:1059-61.

Hegener P, Troke PF, Fatkenheuer G, et al. 1998. Treatment of fluconazoleresistant candidiasis with voriconazole in patients with AIDS. AIDS, 12:2227-8.

Heitmann L, Cometta A, Hurni M, et al. 1997. Right-sided pacemakerrelated endocarditis due to Acremonium species. Clin Infect Dis, 25:158-60.

Herbrecht R. 2004. Voriconazole: therapeutic review of a new azole antifungal. Expert Rev Anti Infect Ther, 2:485-97.

Herbrecht R, Denning DW, Patterson TF, et al. 2002. Voriconazole versus amphotericin B for primary therapy of invasive aspergillosis. $N$ Engl J Med, 347:408-15.

Hernandez PC, Llinares TF, Burgos SJ, et al. 2004. Voriconazole in fungal keratitis caused by Scedosporium apiospermum. Ann Pharmacother, 38:414-17.

Hilliard T, Edwards S, Buchdahl R, et al. 2005. Voriconazole therapy in children with cystic fibrosis. J Cyst Fibros, 4:215-20.

Howden BP, Slavin MA, Schwarer AP, et al. 2003. Successful control of disseminated Scedosporium prolificans infection with a combination of voriconazole and terbinafine. Eur J Clin Microbiol Infect Dis, 22:111-13.

Hsueh PR, Lau YJ, Chuang YC, et al. 2005. Antifungal susceptibilities of clinical isolates of Candida species, Cryptococcus neoformans, and Aspergillus species from Taiwan: surveillance of multicenter antimicrobial resistance in Taiwan program data from 2003. Antimicrob Agents Chemother, 49:512-17.

Husain S, Munoz P, Forrest G, et al. 2005. Infections due to Scedosporium apiospermum and Scedosporium prolificans in transplant recipients: clinical characteristics and impact of antifungal agent therapy on outcome. Clin Infect Dis, 40:89-99. 
Hwang YK, Joo NH, Tee GY, et al. 2001. Clinical activity of the new triazole drug voriconazole (UK 109, 496) against disseminated hepatosplenic aspergillosis in a patient with relapsed leukemia. Haematologia (Budap), 31:73-80.

Imhof A, Balajee SA, Fredricks DN, et al. 2004. Breakthrough fungal infections in stem cell transplant recipients receiving voriconazole. Clin Infect Dis, 39:743-6.

Jabado N, Casanova JL, Haddad E, et al. 1998. Invasive pulmonary infection due to Scedosporium apiospermum in two children with chronic granulomatous disease. Clin Infect Dis, 27:1437-41.

Jang GJ, Kim KS, Shin WS, et al. 2005. Treatment of Candida chorioretinitis with voriconazole. Korean J Ophthalmol, 19:73-6.

Jeu L, Piacenti FJ, Lyakhovetskiy AG, et al. 2003. Voriconazole. Clin Ther, 25:1321-81.

Johnson LB, Kauffman CA. 2003. Voriconazole: a new triazole antifungal agent. Clin Infect Dis, 36:630-7.

Kalenic S, Jandrlic M, Vegar V, et al. 2001. Hansenula anomala outbreak at a surgical intensive care unit: a search for risk factors. Eur $J$ Epidemiol, 17:491-6.

Kanafani ZA, Comair Y, Kan, SS. 2004. Pseudallescheria boydii cranial osteomyelitis and subdural empyema successfully treated with voriconazole: a case report and literature review. Eur J Clin Microbiol Infect Dis, 23:836-40.

Kauffman CA. 2004. Zygomycosis: reemergence of an old pathogen. Clin Infect Dis, 39:588-90.

Khazzam M, Bansal M, Fealy S. 2005. Candida infection of the subacromial bursa. A case report. J Bone Joint Surg Am, 87:168-71.

Kirkpatrick WR, McAtee RK, Fothergill AW, et al. 2000. Efficacy of voriconazole in a guinea pig model of disseminated invasive aspergillosis. Antimicrob Agents Chemother, 44:2865-8.

Kirkpatrick WR, Perea S, Coco BJ, et al. 2002. Efficacy of caspofungin alone and in combination with voriconazole in a guinea pig model of invasive aspergillosis. Antimicrob Agents Chemother, 46:2564-8.

Klasko RK. 2005. Itraconazole/Fluconazole. Drug evaluation monograph. In: Klasko RK (ed). Drugdex ${ }^{\circledR}$ system. Vol 125. Greenwood Village, CO: Thomson Micromedex. (Edition expires 09/2005).

Klastersky J. 2004. Antifungal therapy in patients with fever and neutropenia-more rational and less empirical? $N$ Engl $J$ Med, 351:1445-7.

Kleinpeter MA. 2004. Successful treatment of Candida infections in peritoneal dialysis patients: case reports and review of the literature. Adv Perit Dial, 20:58-61.

Klopfenstein KJ, Rosselet R, Termuhlen A, et al. 2003. Successful treatment of Scedosporium pneumonia with voriconazole during AML therapy and bone marrow transplantation. Med Pediatr Oncol, 41:4945.

Kobayashi K, Kami M, Murashige N, et al. 2004. Breakthrough zygomycosis during voriconazole treatment for invasive aspergillosis. Haematologica, 89:ECR42.

Kontoyiannis DP, Lewis RE. 2004. Toward more effective antifungal therapy: the prospects of combination therapy. $\mathrm{Br} J$ Haematol, 126:165-75.

Kontoyiannis DP, Lionakis MS, Lewis RE, et al. 2005. Zygomycosis in a tertiary-care cancer center in the era of Aspergillus-active antifungal therapy: a case-control observational study of 27 recent cases. J Infect Dis, 191:1350-60.

Kullberg BJ, Sobel JD, Ruhnke M, et al. 2005. Voriconazole versus a regimen of amphotericin B followed by fluconazole for candidaemia in non-neutropenic patients: a randomised non-inferiority trial. Lancet, 366:1435-42.

La Nasa G, Littera R, Maccioni A, et al. 2004. Voriconazole for the treatment of disseminated nodular cutaneous aspergillosis in a patient affected by acute myeloid leukemia. Hematol $J, 5: 178-80$.

Lacroix C, Feuilhade C. 2005. In vitro activities of amphotericin B, itraconazole, voriconazole and caspofungin against Scytalidium dimidiatum and Scytalidium hyalinum isolates. Mycoses, 48(Suppl 2):51.
Lass-Florl C, Fille M, Gunsilius E, et al. 2004. Disseminated infection with Prototheca zopfii after unrelated stem cell transplantation for leukemia. J Clin Microbiol, 42:4907-8.

Lassaletta A, Perez A, Diaz MA, et al. 2004. Successful treatment of invasive aspergillosis with oral voriconazole following intravenous liposomal amphotericin in a child with acute lymphoblastic leukemia. J Pediatr Hematol Oncol, 26:117-19.

Levin T, Suh B, Beltramo D, et al. 2004. Aspergillus mediastinitis following orthotopic heart transplantation: case report and review of the literature. Transpl Infect Dis, 6:129-31.

Linares MJ, Charriel G, Solis F, et al. 2004. Comparison of two microdilution methods for testing susceptibility of Candida spp. to voriconazole. J Clin Microbiol, 42:899-902.

Linares MJ, Charriel G, Solis F, et al. 2005. Susceptibility of filamentous fungi to voriconazole tested by two microdilution methods. $J$ Clin Microbiol, 43:250-3.

Linares MJ, Solis F, Casal, M. 2005. In vitro activity of voriconazole against Prototheca wickerhamii: comparative evaluation of Sensititre and NCCLS M27-A2 methods of detection. J Clin Microbiol, 43:2520-2.

Lutsar I, Roffey S, Troke P. 2003. Voriconazole concentrations in the cerebrospinal fluid and brain tissue of guinea pigs and immunocompromised patients. Clin Infect Dis, 37:728-32.

Maesaki S, Iwakawa J, Higashiyama Y, et al. 2000. Antifungal activity of a new triazole, voriconazole (UK-109496), against clinical isolates of Aspergillus spp. J Infect Chemother, 6:101-3.

Manavathu EK, Cutright JL, Chandrasekar PH. 1998. Organism-dependent fungicidal activities of azoles. Antimicrob Agents Chemother, 42:3018-21.

Marbello L, Nosari A, Carrafiello G, et al. 2003. Successful treatment with voriconazole of cerebral aspergillosis in an hematologic patient. Haematologica, 88:ECR05.

Marco F, Danes C, Almela M, et al. 2003. Trends in frequency and in vitro susceptibilities to antifungal agents, including voriconazole and anidulafungin, of Candida bloodstream isolates. Results from a sixyear study (1996-2001). Diagn Microbiol Infect Dis, 46:259-64.

Marco F, Pfaller MA, Messer SA, et al. 1998. Antifungal activity of a new triazole, voriconazole (UK-109,496), compared with three other antifungal agents tested against clinical isolates of filamentous fungi. Med Mycol, 36:433-6.

Marr KA, Boeckh M, Carter RA, et al. 2004. Combination antifungal therapy for invasive aspergillosis. Clin Infect Dis, 39:797-802.

Martin CA, Roberts S, Greenberg RN. 2002. Voriconazole treatment of disseminated Paecilomyces infection in a patient with acquired immunodeficiency syndrome. Clin Infect Dis, 35:e78-81.

Martin MV, Yates J, Hitchcock CA. 1997. Comparison of voriconazole (UK-109,496) and itraconazole in prevention and treatment of Aspergillus fumigatus endocarditis in guinea pigs. Antimicrob Agents Chemother, 41:13-16.

Marty FM, Cosimi LA, Baden LR. 2004. Breakthrough zygomycosis after voriconazole treatment in recipients of hematopoietic stem-cell transplants. N Engl J Med, 350:950-2.

Mattei D, Mordini N, Lo NC, et al. 2003. Successful treatment of Acremonium fungemia with voriconazole. Mycoses, 46:511-14.

Mattei D, Mordini N, Lo NC, et al. 2002. Voriconazole in the management of invasive aspergillosis in two patients with acute myeloid leukemia undergoing stem cell transplantation. Bone Marrow Transplant, 30:967-70.

Mattner F, Weissbrodt H, Strueber M. 2004. Two case reports: fatal Absidia corymbifera pulmonary tract infection in the first postoperative phase of a lung transplant patient receiving voriconazole prophylaxis, and transient bronchial Absidia corymbifera colonization in a lung transplant patient. Scand J Infect Dis, 36:312-14.

Maxwell MJ, Messer SA, Hollis RJ, et al. 2003. Evaluation of Etest method for determining fluconazole and voriconazole MICs for 279 clinical isolates of Candida species infrequently isolated from blood. $J$ Clin Microbiol, 41:1087-90. 
McGinnis MR, Pasarell L, Sutton DA, et al. 1998. In vitro activity of voriconazole against selected fungi. Med Mycol, 36:239-42.

Meletiadis J, Meis JF, Mouton JW, et al. 2002. In vitro activities of new and conventional antifungal agents against clinical Scedosporium isolates. Antimicrob Agents Chemother, 46:62-8.

Minassian B, Huczko E, Washo T, et al. 2003. In vitro activity of ravuconazole against Zygomycetes, Scedosporium and Fusarium isolates. Clin Microbiol Infect, 9:1250-2.

Moling O, Lass-Floerl C, Verweij PE, et al. 2002. Case reports. Chronic and acute Aspergillus meningitis. Mycoses, 45:504-11.

Montejo M, Muñiz ML, Zárraga S, et al. 2002. Case reports. Infection due to Scedosporium apiospermum in renal transplant recipients: a report of two cases and literature review of central nervous system and cutaneous infections by Pseudallescheria boydii/Sc. apiospermum. Mycoses, 45:418-27.

Mouas H, Lutsar I, Dupont B, et al. 2005. Voriconazole for invasive bone aspergillosis: a worldwide experience of 20 cases. Clin Infect Dis, 40:1141-7.

Muijsers RB, Goa KL, Scott LJ. 2002. Voriconazole in the treatment of invasive aspergillosis. Drugs, 62:2655-64.

Muldrew KM, Maples HD, Stowe CD, et al. 2005. Intravenous voriconazole therapy in a preterm infant. Pharmacotherapy, 25:893-8.

Munoz P, Marin M, Tornero P, et al. 2000. Successful outcome of Scedosporium apiospermum disseminated infection treated with voriconazole in a patient receiving corticosteroid therapy. Clin Infect Dis, 31:1499-501.

Munoz P, Sanchez-Somolinos M, Alcala L, et al. 2005. Candida krusei fungaemia: antifungal susceptibility and clinical presentation of an uncommon entity during 15 years in a single general hospital. $J$ Antimicrob Chemother, 55:188-93.

Murphy M, Bernard EM, Ishimaru T, et al. 1997. Activity of voriconazole (UK-109,496) against clinical isolates of Aspergillus species and its effectiveness in an experimental model of invasive pulmonary aspergillosis. Antimicrob Agents Chemother, 41:696-8.

Nesky MA, McDougal EC, Peacock Jr JE. 2000. Pseudallescheria boydii brain abscess successfully treated with voriconazole and surgical drainage: case report and literature review of central nervous system Pseudallescheriasis. Clin Infect Dis, 31:673-7.

Nulens E, Eggink C, Rijs AJ, et al. 2003. Keratitis caused by Scedosporium apiospermum successfully treated with a cornea transplant and voriconazole. J Clin Microbiol, 41:2261-4.

Oren I. 2005. Breakthrough zygomycosis during empirical voriconazole therapy in febrile patients with neutropenia. Clin Infect Dis, 40:770-1.

Ostrosky-Zeichner L, Oude Lashof AM, Kullberg BJ, et al. 2003. Voriconazole salvage treatment of invasive candidiasis. Eur J Clin Microbiol Infect Dis, 22:651-5.

Ostrosky-Zeichner L, Rex JH, Pappas PG, et al. 2003. Antifungal susceptibility survey of 2,000 bloodstream Candida isolates in the United States. Antimicrob Agents Chemother, 47:3149-54.

Pai MP, Jones AL. 2004. Altered susceptibility of Candida glabrata bloodstream isolates to triazoles at clinically relevant $\mathrm{pH}$ values: comparison of the NCCLS M27-A2, Sensititre YeastOne, and Etest methods. Antimicrob Agents Chemother, 48:4441-3.

Paphitou NI, Ostrosky-Zeichner L, Paetznick VL, et al. 2002. In vitro antifungal susceptibilities of Trichosporon species. Antimicrob Agents Chemother, 46:1144-6.

Pearson MM, Rogers PD, Cleary JD, et al. 2003. Voriconazole: a new triazole antifungal agent. Ann Pharmacother, 37:420-32.

Pelletier R, Loranger L, Marcotte H, et al. 2002. Voriconazole and fluconazole susceptibility of Candida isolates. J Med Microbiol, 51:479-83.

Pemán J, Cantón E, Gobernado M. 2005. Epidemiology and antifungal susceptibility of Candida species isolated from blood: results of a 2year multicentre study in Spain. Eur J Clin Microbiol Infect Dis, 24:23-30.
Perea S, Gonzalez G, Fothergill AW, et al. 2002. In vitro interaction of caspofungin acetate with voriconazole against clinical isolates of Aspergillus spp. Antimicrob Agents Chemother, 46:3039-41.

Perfect JR, Marr KA, Walsh TJ, et al. 2003. Voriconazole treatment for less-common, emerging, or refractory fungal infections. Clin Infect Dis, 36:1122-31.

Perlroth MG, Miller J. 2004. Pseudallescheria boydii pneumonia and empyema: a rare complication of heart transplantation cured with voriconazole. J Heart Lung Transplant, 23:647-9.

Petit A, Tabone MD, Moissenet D, et al. 2005. Disseminated Fusarium infection in two neutropenic children. Arch Pediatr, 12:1116-19.

Pfaller MA, Jones RN, Doern GV, et al. 1999. International surveillance of blood stream infections due to Candida species in the European SENTRY Program: species distribution and antifungal susceptibility including the investigational triazole and echinocandin agents. SENTRY Participant Group (Europe). Diagn Microbiol Infect Dis, 35:19-25.

Pfaller MA, Messer SA, Gee S, et al. 1999. In vitro susceptibilities of Candida dubliniensis isolates tested against the new triazole and echinocandin antifungal agents. J Clin Microbiol, 37:870-2.

Pfaller MA, Messer SA, Hollis RJ, et al. 2002a. Antifungal activities of posaconazole, ravuconazole, and voriconazole compared to those of itraconazole and amphotericin B against 239 clinical isolates of Aspergillus spp. and other filamentous fungi: report from SENTRY Antimicrobial Surveillance Program, 2000. Antimicrob Agents Chemother, 46:1032-7.

Pfaller MA, Messer SA, Hollis RJ, et al. 2002b. In vitro activities of ravuconazole and voriconazole compared with those of four approved systemic antifungal agents against 6,970 clinical isolates of Candida spp. Antimicrob Agents Chemother, 46:1723-1727.

Pfaller MA, Diekema DJ, Messer SA, et al. 2003. In vitro activities of voriconazole, posaconazole, and four licensed systemic antifungal agents against Candida species infrequently isolated from blood. $J$ Clin Microbiol, 41:78-83.

Pfaller MA, Diekema DJ. 2004. Rare and emerging opportunistic fungal pathogens: concern for resistance beyond Candida albicans and Aspergillus fumigatus. J Clin Microbiol, 42:4419-31.

Pfaller MA, Espinel-Ingroff A, Jones RN. 2004. Clinical evaluation of the Sensititre YeastOne colorimetric antifungal plate for antifungal susceptibility testing of the new triazoles voriconazole, posaconazole, and ravuconazole. J Clin Microbiol, 42:4577-80.

Pfaller MA, Messer SA, Boyken L, et al. 2004. In vitro activities of voriconazole, posaconazole, and fluconazole against 4,169 clinical isolates of Candida spp. and Cryptococcus neoformans collected during 2001 and 2002 in the ARTEMIS global antifungal surveillance program. Diagn Microbiol Infect Dis, 48:201-5.

Pfaller MA, Boyken L, Messer SA, et al. 2005. Comparison of results of voriconazole disk diffusion testing for Candida species with results from a central reference laboratory in the ARTEMIS global antifungal surveillance program. J Clin Microbiol, 43:5208-13.

Pfaller MA, Messer SA, Boyken L, et al. 2005. Global trends in the antifungal susceptibility of Cryptococcus neoformans (1990 to 2004). J Clin Microbiol, 43:2163-7.

Polizzi A, Siniscalchi C, Mastromarino A, et al. 2004. Effect of voriconazole on a corneal abscess caused by Fusarium. Acta Ophthalmol Scand, 82:762-4.

Poza G, Montoya J, Redondo C, et al. 2000. Meningitis caused by Pseudallescheria boydii treated with voriconazole. Clin Infect Dis, 30:981-2.

Prabhu RM, Bonnell M, Currier BL, et al. 2004. Successful treatment of disseminated nonmeningeal coccidioidomycosis with voriconazole. Clin Infect Dis, 39:e74-7.

Purkins L, Wood N, Kleinermans D, et al. 2003. Voriconazole potentiates warfarin-induced prothrombin time prolongation. $\mathrm{Br} J \mathrm{Clin}$ Pharmacol, 56(Suppl 1):24-9. 
Puzniak L, Teutsch S, Powderly W, et al. 2004. Has the epidemiology of nosocomial candidemia changed? Infect Control Hosp Epidemiol, 25:628-33.

Quindós G, Carrillo-Muñoz AJ, Arévalo MP, et al. 2000. In vitro susceptibility of Candida dubliniensis to current and new antifungal agents. Chemotherapy, 46:395-401.

Radford SA, Johnson EM, Warnock DW. 1997. In vitro studies of activity of voriconazole (UK-109,496), a new triazole antifungal agent, against emerging and less-common mold pathogens. Antimicrob Agents Chemother, 41:841-3.

Reis A, Sundmacher R, Tintelnot K, et al. 2000. Successful treatment of ocular invasive mould infection (fusariosis) with the new antifungal agent voriconazole. Br J Ophthalmol, 84:932-3.

Reis LJ, Barton TD, Pochettino A, et al. 2005. Successful treatment of Aspergillus prosthetic valve endocarditis with oral voriconazole. Clin Infect Dis, 41:752-3.

Rodriguez CA, Lujan-Zilbermann J, Woodard P, et al. 2003. Successful treatment of disseminated fusariosis. Bone Marrow Transplant, $31: 411-12$.

Rodriguez-Tudela JL, Diaz-Guerra TM, Mellado E, et al. 2005. Susceptibility patterns and molecular identification of Trichosporon species. Antimicrob Agents Chemother, 49:4026-34.

Rosen-Wolff A, Koch A, Friedrich W, et al. 2004. Successful elimination of an invasive Aspergillus nidulans lung infection by voriconazole after failure of a combination of caspofungin and liposomal amphotericin B in a boy with chronic granulomatous disease. Pediatr Infect Dis $J, 23: 584-6$.

Rubio MC, Gil J, Ramírez de Ocariz I, et al. 2003. In vitro activity of fluconazole, voriconazole and posaconazole against Candida spp. Rev Esp Quimioter, 16:227-32.

Rubio MC, Ramírez de Ocariz IR, Gil J, et al. 2005. Potential fungicidal effect of voriconazole against Candida spp. Int J Antimicrob Agents, 25:264-7.

Sabo JA, Abdel-Rahman SM. 2000. Voriconazole: a new triazole antifungal. Ann Pharmacother, 34:1032-43.

Sadaba B, Campanero MA, Quetglas EG, et al. 2004. Clinical relevance of sirolimus drug interactions in transplant patients. Transplant Proc, $36: 3226-8$.

Sambatakou H, Guiver M, Denning D. 2003. Pulmonary aspergillosis in a patient with chronic granulomatous disease: confirmation by polymerase chain reaction and serological tests, and successful treatment with voriconazole. Eur J Clin Microbiol Infect Dis, 22:6815.

Schaenman JM, Digiulio DB, Mirels LF, et al. 2005. Scedosporium apiospermum soft tissue infection successfully treated with voriconazole: potential pitfalls in the transition from intravenous to oral therapy. J Clin Microbiol, 43:973-7.

Schlamm H, Corey L, Brown J, et al. 2000. Voriconazole for salvage treatment of invasive aspergillosis [abstract]. Clin Infect Dis, 31:265.

Schlamm H, Supparatpnyo K. 2003. Voriconazole as therapy for systemic infections caused by Penicillium marneffei in patients with HIV infection [abstract]. 43rd Interscience Conference on Antimicrobial Agents Chemotherapy, September 14-17, Chicago, IL, USA. M-963.

Schwartz S, Ruhnke M, Ribaud P, et al. 2005. Improved outcome in central nervous system aspergillosis with voriconazole treatment. Blood, 106:2641-5.

Serrano MC, Ramirez M, Morilla D, et al. 2004. A comparative study of the disc diffusion method with the broth microdilution and Etest methods for voriconazole susceptibility testing of Aspergillus spp. $J$ Antimicrob Chemother, 53:739-42.

Serrano MC, Valverde-Conde A, Chavez MM, et al. 2003. In vitro activity of voriconazole, itraconazole, caspofungin, anidulafungin (VER002, LY303366) and amphotericin B against Aspergillus spp. Diagn Microbiol Infect Dis, 45:131-5.
Serrano-Martino MC, Chavez-Caballero M, Valverde-Conde A, et al. 2003. In vitro activity of voriconazole and three other antifungal agents against dermatophytes. Enferm Infecc Microbiol Clin, 21:484-7.

Shouldice E, Fernandez C, McCully B, et al. 2003. Voriconazole treatment of presumptive disseminated Aspergillus infection in a child with acute leukemia. J Pediatr Hematol Oncol, 25:732-4.

Siwek GT, Dodgson KJ, Magalhaes-Silverman M, et al. 2004. Invasive zygomycosis in hematopoietic stem cell transplant recipients receiving voriconazole prophylaxis. Clin Infect Dis, 39:584-7.

Steinbach WJ. 2005. Antifungal agents in children. Pediatr Clin North Am, 52:895-915.

Steinbach WJ, Benjamin DK Jr, Kontoyiannis DP, et al. 2004. Infections due to Aspergillus terreus: a multicenter retrospective analysis of 83 cases. Clin Infect Dis, 39:192-8.

Steinbach WJ, Schell WA, Miller JL, et al. 2003. Scedosporium prolificans osteomyelitis in an immunocompetent child treated with voriconazole and caspofungin, as well as locally applied polyhexamethylene biguanide. J Clin Microbiol, 41:3981-5.

Stern JB, Girard P, Caliandro R. 2004. Pleural diffusion of voriconazole in a patient with Aspergillus fumigatus empyema thoracis. Antimicrob Agents Chemother, 48:1065.

Stratov I, Korman TM, Johnson PD. 2003. Management of Aspergillus osteomyelitis: report of failure of liposomal amphotericin B and response to voriconazole in an immunocompetent host and literature review. Eur J Clin Microbiol Infect Dis, 22:277-83.

Studahl M, Backteman T, Stalhammar F, et al. 2003. Bone and joint infection after traumatic implantation of Scedosporium prolificans treated with voriconazole and surgery. Acta Paediatr, 92:980-2.

Sugar AM, Liu XP. 2001. Efficacy of voriconazole in treatment of murine pulmonary blastomycosis. Antimicrob Agents Chemother, 45:601-4.

Swift AC, Denning DW. 1998. Skull base osteitis following fungal sinusitis. J Laryngol Otol, 112:92-7.

Swinne D, Watelle M, Nolard N. 2005. In vitro activities of voriconazole, fluconazole, itraconazole and amphotericin B against non-Candida albicans yeast isolates. Rev Iberoam Micol, 22:24-8.

Swinne D, Watelle M, van der Flaes M, et al. 2004. In vitro activities of voriconazole (UK-109, 496), fluconazole, itraconazole and amphotericin B against 132 non-albicans bloodstream yeast isolates (CANARI study). Mycoses, 47:177-83.

Takakura S, Fujihara N, Saito T, et al. 2004. National surveillance of species distribution in blood isolates of Candida species in Japan and their susceptibility to six antifungal agents including voriconazole and micafungin. J Antimicrob Chemother, 53:283-9.

Tattevin P, Bruneel F, Lellouche F, et al. 2004. Successful treatment of brain aspergillosis with voriconazole. Clin Microbiol Infect, 10:928-31.

Tortorano AM, Pemán J, Bernhardt H, et al. 2004. Epidemiology of candidaemia in Europe: results of 28-month European Confederation of Medical Mycology (ECMM) hospital-based surveillance study. Eur $J$ Clin Microbiol Infect Dis, 23:317-22.

Tortorano AM, Rigoni AL, Biraghi E, et al. 2003. The European Confederation of Medical Mycology (ECMM) survey of candidaemia in Italy: antifungal susceptibility patterns of 261 non-albicans Candida isolates from blood. J Antimicrob Chemother, 52:679-82.

Trinh JV, Steinbach WJ, Schell WA, et al. 2003. Cerebral phaeohyphomycosis in an immunodeficient child treated medically with combination antifungal therapy. Med Mycol, 41:339-45.

Troke P, Schwartz S, Ruhnke M, et al. 2003. Voriconazole therapy in 86 patients with CNS aspergillosis: a retrospective analysis [abstract]. 43th Interscience Conference on Antimicrobial Agents and Chemotherapy. September 14-17, Chicago, IL, USA. M-1755.

Uzun O, Arikan S, Kocagoz S, et al. 2000. Susceptibility testing of voriconazole, fluconazole, itraconazole and amphotericin B against yeast isolates in a Turkish University Hospital and effect of time of reading. Diagn Microbiol Infect Dis, 38:101-7. 
van Duin D, Cleare W, Zaragoza O, et al. 2004. Effects of voriconazole on Cryptococcus neoformans. Antimicrob Agents Chemother, 48:2014-20.

Varma D, Thaker HR, Moss PJ, et al. 2005. Use of voriconazole in candida retinitis. Eye, 19:485-7.

Vazquez JA, Lynch M, Boikov D, et al. 1997. In vitro activity of a new pneumocandin antifungal, L-743,872, against azole-susceptible and resistant Candida species. Antimicrob Agents Chemother, 41:1612-14.

Venkataramanan R, Zang S, Gayowski T, et al. 2002. Voriconazole inhibition of the metabolism of tacrolimus in a liver transplant recipient and in human liver microsomes. Antimicrob Agents Chemother, 46:3091-3.

Verweij PE, van den Bergh MF, Rath PM, et al. 1999. Invasive aspergillosis caused by Aspergillus ustus: case report and review. J Clin Microbiol, 37:1606-9.
Vigouroux S, Morin O, Moreau P, et al. 2005. Zygomycosis after prolonged use of voriconazole in immunocompromised patients with hematologic disease: attention required. Clin Infect Dis, 40:e35-7.

Vincent AL, Cabrero JE, Greene JN, et al. 2003. Successful voriconazole therapy of disseminated Fusarium solani in the brain of a neutropenic cancer patient. Cancer Control, 10:414-19.

Walsh TJ, Lutsar I, Driscoll T, et al. 2002. Voriconazole in the treatment of aspergillosis, scedosporiosis and other invasive fungal infections in children. Pediatr Infect Dis J, 21:240-8.

Walsh TJ, Pappas P, Winston DJ, et al. 2002. Voriconazole compared with liposomal amphotericin B for empirical antifungal therapy in patients with neutropenia and persistent fever. $N$ Engl J Med, 346:225-34.

Yang YL, Cheng HH, Lo HJ. 2004. In vitro activity of voriconazole against Candida species isolated in Taiwan. Int J Antimicrob Agents, 24:294-6. 
